\title{
Low Complexity Dynamic Channel Equalization in OFDM with High Frequency Mobile Network Technologies
}

\author{
Redhwan Mawari, Mohamed Zohdy \\ Department of Electrical and Computer Engineering, Oakland University, Rocheser, MI, USA \\ Email: rmawari@oakland.edu, zohdyma@oakland.edu
}

How to cite this paper: Mawari, R. and Zohdy, M. (2017) Low Complexity Dynamic Channel Equalization in OFDM with High Frequency Mobile Network Technologies. Journal of Signal and Information Processing, 8, 17-41.

https://doi.org/10.4236/jsip.2017.82003

Received: March 26, 2017

Accepted: May 2, 2017

Published: May 5, 2017

Copyright ( 92017 by authors and Scientific Research Publishing Inc. This work is licensed under the Creative Commons Attribution International License (CC BY 4.0).

http://creativecommons.org/licenses/by/4.0/

\begin{abstract}
We present in this paper a method for enhancing equalization of a dynamic channel. A dynamic channel is characterized and modeled by a high relative velocity between transmitter and receiver and fast changes of environment conditions for wave propagation. Based on Jakes model, an auto-regressive model (AR) [1] for such a dynamic system, i.e., a time variant channel is developed. More specifically, the enhanced equalization method we are proposing is a combination of a multi-stage time and frequency domain equalizer with a feed-forward loop. The underlined wok presents a unified approach to the equalization method that employs both time and frequency domains data to enhance the equalization scheme. In an OFDM system, the channel coefficients for each tap, in time domain for consecutive blocks, are partially independent thus correlated. Such correlation can improve the channel estimation if it is taken into account. The method in this paper enhances the performance of equalization by dynamically selecting the number of previous OFDM symbols based on the Doppler frequency. In order to decrease the complexity of the system model, we utilize the autocorrelation and Doppler frequency to dynamically select the previous OFDM symbols that will be stored in the memory. In addition to deriving earlier results in a unified manner, the approach presented also leads to enhanced performance results without imposing any restrictions or limitations on the OFDM system such as increasing the number of pilots or cyclic prefix.
\end{abstract}

\section{Keywords}

Equalization, Kalman, Auto-Regressive, OFDM, Jakes Model

\section{Introduction}

There is much on-going research in channel estimation procedures, however, 
most of it considers a time-invariant channel [2]. The OFDM system renders the estimation process less computationally complex, but dramatically degrades the performance at different mobile speeds or low Signal-to-Noise Ratios (SNRs). In order to accurately characterize the channel under more realistic conditions and to validate the best fit models, $2 \mathrm{D}$ testing was conducted on a mobile receiver under various conditions [3]. The results of the testing were close enough to the models of the channel. However, when increasing the spectrum of the real life scenarios such as the receiver moving at different speeds, a Doppler shift occurs. Thus, the mobility results are in a Doppler time variant and fast fading channel. Furthermore, reflection off nearby objects can lead to multi-path signals which can result into frequency selective fading due to destructive interference. In order to overcome such concerns, the OFDM system [4] as shown in Figure 1, and also further illustrated more in details in a patent publication by [5], divides the channel into narrowband flat fading sub-channels, consequently, the OFDM system is more resistant to frequency selective fading than single carrier systems are. Furthermore, the OFDM system eliminates Inter-symbol Interference (ISI) and Inter-Carrier Interference (ICI) through the use of a cyclic prefix. Thus, one of the advantages of the OFDM is that channel equalization becomes simpler than using adaptive equalization techniques with single carrier systems. OFDM is computationally efficient by using FFT techniques [6] to implement the modulation and demodulation functions. There has been a tremendous amount of work on modulation/demodulation optimization using different types of estimators such as a Kalman filter [7] [8]. The OFDM system forms the basis for $4 \mathrm{G}$ wireless communication systems. Long Term Evolution (LTE) is $4^{\text {th }}$ cellular generation standard based on OFDM. The OFDM system is a key broadband wireless technology which supports data rates in excess of $100 \mathrm{Mbps}$.

Therefore, channel estimation, channel equalization, and the iterative complex algorithms would suffer from high complexity as well as poor performance over fast fading frequency selective channels [9]. Some alternative solutions have been proposed either to decrease the complexity or to enhance the performance [10] [11], but the performance gain is not evident to one ordinary skilled in the

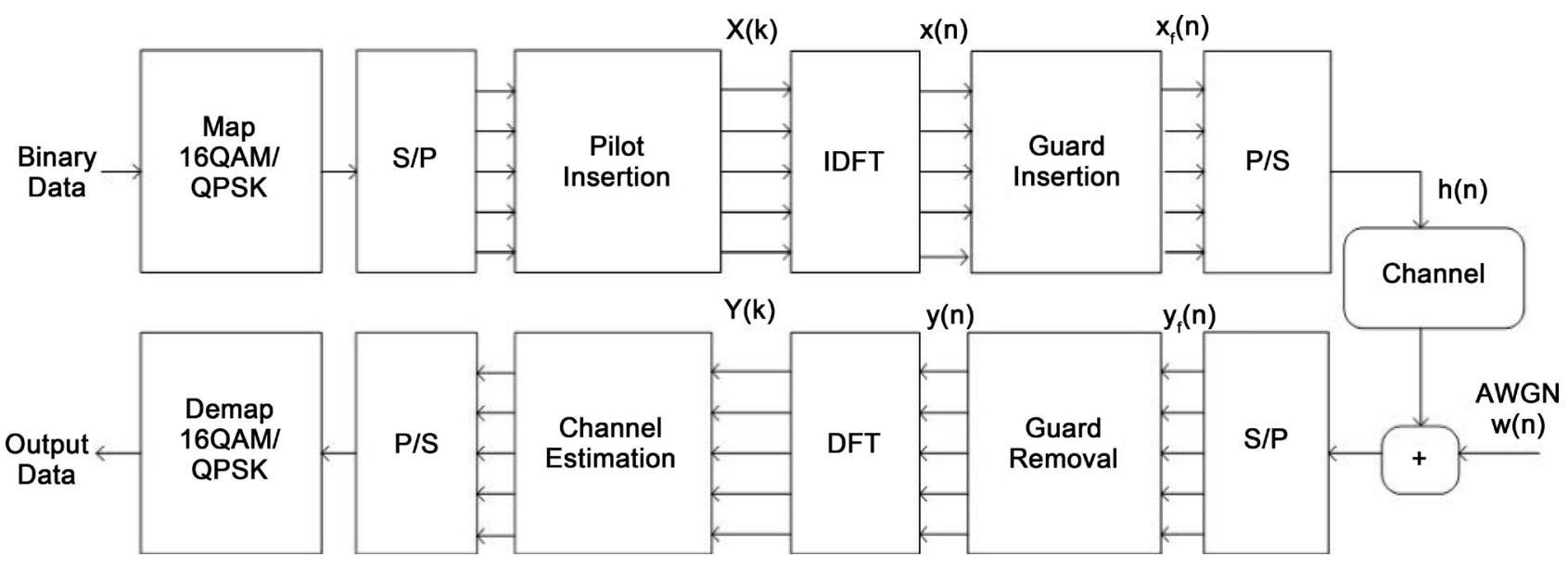

Figure 1. OFDM system structure. 
field. One solution to combat the interference is to add the cyclic prefix [12], in the OFDM scheme, however, the addition of the cyclic prefix does not resolve the interference issue to an acceptable and satisfactory performance. In order to mitigate ISI and ICI, the Cyclic prefix length must be more than or equal to the channel impulse response (CIR), otherwise the system suffers from insufficient cyclic prefix distortion [13]. However, even if increasing cyclic prefix would improve the performance of the system, the effect of the addition of long CP causes a loss in throughput of the system as well as loss in efficiency. Thus, the complex algorithm with the deteriorated performance over fast time-varying channels is still required for channel estimation and OFDM equalization [14]. Furthermore, increasing the inserted pilot in the OFDM symbols will result into a much higher average power than the normal OFDM system data [15], thus the equivalent signal-to-noise ratio (SNR) at the receiver will be reduced. The OFDM system eliminates (ISI) and (ICI) through the use of a cyclic prefix. However, in a high speed mobility and higher frequency mobile network technologies such as but not limited to $4 \mathrm{G}$, where a wireless signal propagates over rapidly varying channels occurring in high mobility scenarios, a frequency selective phenomenon occurs. As a result, the OFDM system is not resistant to frequency selective fading and thus the narrowband channels are not flat anymore. In order to eliminate ICI and ISI in an OFDM system with high mobility scenarios, an increase in a prefix value is necessary and thus the energy consumption will increase. An OFDM system can have adaptive frequency equalizers such as decision-feedback equalizers [15] which are exploited to reduce the ICI and ISI, however, equalizers such as Decision-feedback equalizers are computationally complex and can introduce errors when wrong decisions are made. As shown in the left side of Figure 2, and also illustrated more in details by [16], the channel is at and thus the signal is distorted. However, on the right side, the channel experiences a deep fading at certain points and thus the channel response is affected by this phenomenon. Even though the OFDM attempts to resolve the frequency selectivity issue, which it does to a certain point, there are still frequency selectivity issues the OFDM system is not able to resolve. Figure 3, as illustrated more in details by [16], shows that there are some subcarriers that are affected by deep fading. Thus, OFDM system alone does not work under more critical conditions.

We assume high frequency mobile network technologies, such as but not limited to $4 \mathrm{G}$, and a wireless signal that propagates over rapidly varying channels

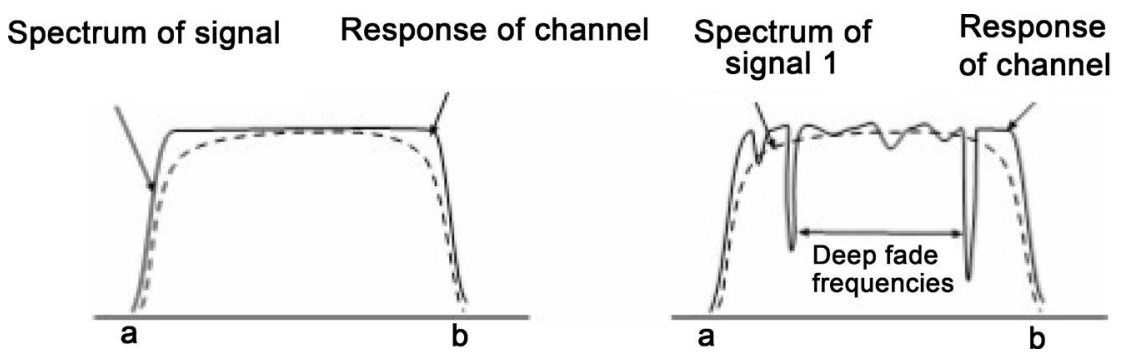

Figure 2. Frequency flat fading versus frequency selective fading. 
Subcarriers effected by fades

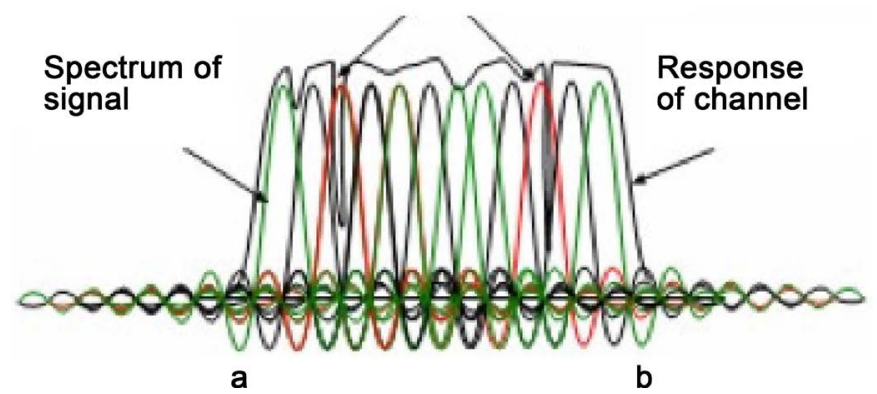

Figure 3. Frequency selective fading in OFDM system.

occurring at relatively high speeds. Thus, based on the set forth conditions of the channel, we utilize a model known as Jakes model. With Jakes model, the channel is correlated in time due to the Doppler effect. This model enables us to adapt Jakes model for the time varying channel taps, i.e. the underlying channel varies with time. Furthermore, with Jakes model, we are able to estimate the next sample of the channel utilizing the fact that the Auto-regressive model estimates the system based on the previous samples, since the estimation of the next sample of the channel primarily depends on the previous samples. As such, it is obvious that the higher the number of previous samples, the more accurate estimate is obtained. However, the caveat with increasing the number of previous samples is that it causes inefficiency in the system and thus renders this method irrational. Therefore, this paper will introduce the relationship between the number of the previous samples and Doppler shift in which renders the selection of previous number of samples more efficient.

In order to select a precise number of previous OFDM symbols, optimize, and utilize the memory and store said symbols efficiently, this paper takes an advantage of the relationship between the Doppler and the auto-correlation of the subsequent OFDM symbols. This approach will lead to a less complex and more efficient system. The Kalman [17] estimator combines the two independent estimates of the channel. The current data estimate of the channel is combined with the predicted (from the model) estimate. This current estimate is then projected through the channel model to predict the next state of the channel. Both these steps are combined into a concise form by using the Kalman filter. As a result of using Jakes model in conjunction with the data estimates, the Kalman filter based channel estimation procedure drastically improves on the performance of the data-only estimator. We are also able to predict the next state of the channel with an enhanced accuracy based on the number of previous OFDM symbols. More specifically, the enhanced equalization method we are proposing is a combination of a multi-stage time and frequency domain equalizer with a feed-forward loop. Inserting pilot data at the transmitter and using said pilot data to estimate the channel in time domain. The addition of the feed-forward is utilized to capture a part of the received signal, then extracting the channel information while the signal is being delayed until the information is obtained. The estimated channel is forwarded to a Minimum Mean Square Error (MMSE) 
equalizer. The first stage of the multi-stage equalizer is using pilot data as a measurement data in the frequency domain. The second stage is to convert the measurement data to the time domain. In the third stage, we estimate the channel using the pilot data and use it as a measurement data, i.e. input to the Kalman filter. In the OFDM system, the channel coefficients for each tap in time domain for consecutive blocks are not totally independent, but correlated. Such a correlation can improve the channel estimation if it is taken into account. Using Kalman filter, we can extract this information from previous blocks, use it for the newly arrived one, and perform a better channel estimation. The fourth stage, we use the output of the Kalman filter as a feed-forward to the frequency equalizer for each subcarrier in which the data is converted from the time domain to frequency domain.

\section{System Model}

The channel is correlated in time domain due to the Doppler effect. Doppler follows Jakes model where the auto-correlation is not an impulse. Thus, in this case, such a dynamic channel follows Jakes model, and the auto-correlation of Jake model can be easily obtained by the following:

$$
R[n]=J_{0}\left(\pi f_{m} n\right)
$$

where $f_{m}$ is the maximum Doppler frequency, where $J_{0}($.$) is the zeroth-$ order Bessel function. The next sample can be predicted based on the AR process given by:

$$
s(n+1)=\sum_{k=0}^{M-1} \alpha_{k} s(n-k)+w(n)
$$

Based on the assumption that $\alpha_{0}=1$; we can readily derive the Auto- correlation function as follows:

$$
\begin{gathered}
\alpha_{0} R(l-k)+\sum_{k=1}^{M} \alpha_{k} R(l-k)=0 \\
\alpha_{0} R(0-l)+\sum_{k=1}^{M} \alpha_{k} R(l-k)=0 \\
R_{x x}(-l)=-\sum_{k=1}^{M} \alpha_{k} R_{x x}(l-k)
\end{gathered}
$$

wherein $\alpha$ is given by:

$$
\alpha=R_{x x}^{-1} r
$$

This can be written in a matrix form as follows:

$$
R_{x x}=\left[\begin{array}{cccc}
r_{x x}[0] & r_{x x}[-1] & \cdots & r_{x x}[1-M] \\
r_{x x}[1] & r_{x x}[0] & \cdots & r_{x x}[2-M] \\
r_{x x}[2] & r_{x x}[1] & \cdots & r_{x x}[3-M] \\
\vdots & \vdots & \ddots & \vdots \\
r_{x x}[M-1] & r_{x x}[M-2] & \cdots & r_{x x}[0]
\end{array}\right]
$$

where $\alpha$ is written in a vector form: 


$$
\begin{gathered}
\alpha=\left[\alpha_{1}, \alpha_{2}, \cdots, \alpha_{M}\right]^{\mathrm{T}} \\
r=\left[r_{x x}[1], r_{x x}[2], r_{x x}[3], \cdots, r_{x x}[M]\right]
\end{gathered}
$$

Given $\alpha$, we can solve for $\sigma$ as follows:

$$
\sigma^{2}=R_{x x}[0]+\sum_{k=1}^{M} \alpha_{k} R_{x x}[-k]
$$

Now, we can form the system model matrix based on Equations ((2) and (7)) as follows:

$$
\left[\begin{array}{c}
s(n+1) \\
s(n) \\
\vdots \\
s(n-m-1)
\end{array}\right]=\left[\begin{array}{cccc}
\alpha_{0} & \alpha_{1} & \cdots & \alpha_{M-1} \\
1 & 0 & \cdots & 0 \\
0 & 1 & \cdots & 0 \\
0 & 0 & \cdots & 1
\end{array}\right]\left[\begin{array}{c}
s(n) \\
s(n-1) \\
\vdots \\
s(n-m)
\end{array}\right]+\left[\begin{array}{c}
w(n) \\
0 \\
\vdots \\
0
\end{array}\right]
$$

The system model can be written in a vector form as follows:

$$
s^{n+1}=F S^{n}+w
$$

\section{Equalizer}

\subsection{Kalman Filter}

For a single tap channel, the state space model for the $1^{\text {st }}$ tap is given by:

$$
S_{1}^{k}=F S_{1}^{k-1}+Z_{1}
$$

where, $S_{1}$ is the state, $F$ is the transition matrix, $S_{1}^{k-1}$ is the previous state, and $Z_{1}$ is the covariance matrix.

$$
S_{1}^{k}=\left[\begin{array}{c}
h_{1}(k) \\
h_{1}(k-1) \\
h_{1}(k-2) \\
\vdots \\
h_{1}\left(k-N_{s}-1\right)
\end{array}\right]
$$

where $h$ is the channel coefficients at time instant $k$.

The transition matrix $F$ for the $1^{\text {st }}$ tap is given by:

$$
F=\left[\begin{array}{cccc}
\alpha_{0} & \alpha_{1} & \cdots & \alpha_{N_{S}-1} \\
1 & 0 & \cdots & 0 \\
0 & 1 & \cdots & 0 \\
0 & 0 & \cdots & 1
\end{array}\right]
$$

The previous state is given by:

$$
S_{1}^{k-1}=\left[\begin{array}{c}
h_{1}(k-1) \\
h_{1}(k-2) \\
h_{1}(k-3) \\
\vdots \\
h_{1}\left(k-N_{s}\right)
\end{array}\right]
$$

The covariance matrix for the $1^{\text {st }}$ tap is given by: 


$$
Z_{1}=\left[\begin{array}{c}
Z_{1} \\
0 \\
\vdots \\
0
\end{array}\right]
$$

The state space model for the $2^{\text {nd }}$ tap is given by:

$$
S_{2}^{k}=F S_{2}^{k-1}+Z_{2}
$$

Finally, for multiple taps channel, the state space model is given by:

$$
\begin{gathered}
S_{L}^{k}=F S_{L}^{k-1}+Z_{L} \\
{\left[\begin{array}{c}
S_{1}^{k} \\
S_{2}^{k} \\
\vdots \\
S_{L}^{k}
\end{array}\right]=\left[\begin{array}{ccccc}
F & 0 & 0 & \cdots & 0 \\
0 & F & 0 & \cdots & 0 \\
0 & 0 & F & \cdots & 0 \\
\vdots & \vdots & \vdots & \ddots & \vdots \\
0 & 0 & 0 & \cdots & F
\end{array}\right]\left[\begin{array}{c}
S_{1}^{k}-1 \\
S_{2}^{k}-1 \\
\vdots \\
S_{L}^{k}-1
\end{array}\right]+\left[\begin{array}{c}
Z_{1} \\
Z_{2} \\
\vdots \\
Z_{L}
\end{array}\right]}
\end{gathered}
$$

The measurement output of the state space model is as follows:

$$
y^{k}=H S^{k}+n^{k}
$$

In summary, the state space model and the measurement output for the channel is as follows:

$$
\begin{gathered}
S^{k}=F S^{k-1}+Z_{k} \\
y^{k}=H S^{k}+n^{k}
\end{gathered}
$$

Once we calculate $\alpha$, we can readily calculate $F$ matrix.

\subsection{OFDM}

Figure 4 shows a high level representation of the OFDM system. Initially, data is encoded in frequency domain and subsequently using IFFT transformation to

\section{Orthogonal Frequency Division Modulation}
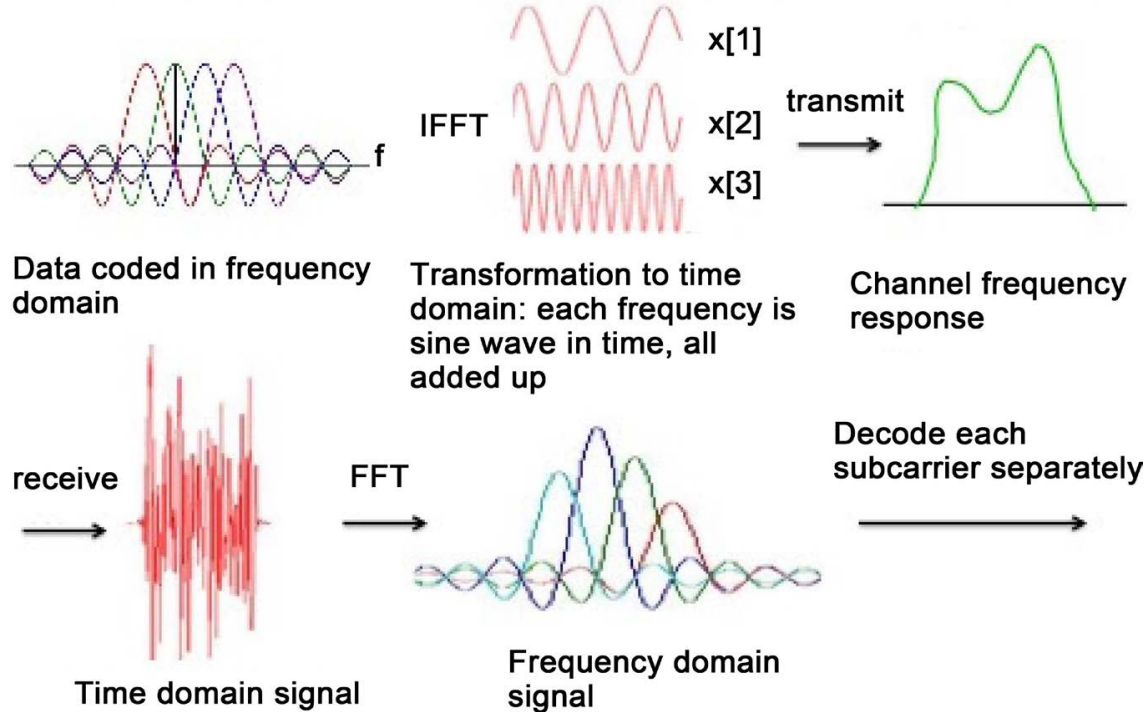

Figure 4. OFDM process. 
transform the data to time domain. Next, data is summed and transmitted to a receiver through a channel. At the receiver, data is in time domain thus it is transformed to frequency domain and then decoded in order to retrieve the original data.

Equation below shows a mathematical model of a composite transmitted and received signals in time and frequency domain as follows:

$$
\begin{aligned}
& X[K]=\sum_{n=0}^{N-1} x(n) \mathrm{e}^{\frac{-j 2 \pi}{N} k n} \\
& x[n]=\sum_{n=0}^{N-1} X(K) \mathrm{e}^{\frac{-j 2 \pi}{N} k n}
\end{aligned}
$$

Both equations defined above represent Discrete Fourier Transform (DFT) which transfers the samples from time domain to frequency domain. The Inverse Discrete Fourier Transform which transfers the frequency domain symbols into time domain samples.

IFFT operation is defined below:

$$
x=F_{N}^{*} X
$$

The time domain $N \times 1$ representation of $X$ which is $N \times 1$ matrix. $F_{N}$ is $N \times N$ matrix containing $F F$ coefficients.

$$
F_{N}=\left[\begin{array}{ccccc}
\mathrm{e}^{0} & \mathrm{e}^{0} & \mathrm{e}^{0} & \cdots & \mathrm{e}^{0} \\
\mathrm{e}^{0} & \mathrm{e}^{-\frac{j 2 \pi}{N}} & \mathrm{e}^{-\frac{j 4 \pi}{N}} & \cdots & \mathrm{e}^{-\frac{j 2 \pi 2(N-1)}{N}} \\
\mathrm{e}^{0} & \mathrm{e}^{-\frac{-j 4 \pi}{N}} & \mathrm{e}^{-\frac{-j 8 \pi}{N}} & \cdots & \mathrm{e}^{-\frac{j 2 \pi 2(N-1)}{N}} \\
\vdots & \vdots & \vdots & \ddots & \vdots \\
\mathrm{e}^{0} & \mathrm{e}^{-\frac{j 2 \pi(N-1)}{N}} & \mathrm{e}^{-\frac{j 2 \pi 2(N-1)}{N}} & \cdots & \mathrm{e}^{-\frac{j 2 \pi 2(N-1)}{N}}
\end{array}\right]
$$

A cyclic prefix refers to the prefixing of a symbol with a repetition of the end. The prefix serves as a guard interval, it eliminates the inter-symbol interference from the previous symbol. Also, the prefix serves as a repetition of the end of the symbol, it allows the linear convolution of a frequency-selective multipath channel to be modelled as circular convolution, which in turn may be transformed to the frequency domain using a discrete Fourier transform. This approach allows for simple frequency-domain processing, such as channel estimation and equalization.

Cyclic Prefixes are used in OFDM in order to combat multipath by making channel estimation easy. As an example, consider an OFDM system which has $\mathrm{N}$ subcarriers.

A cyclic prefix (CP) of length is added to the time domain samples to make a more complete OFDM symbol or packet with samples. As mentioned, this cyclic prefix preserves orthogonality between the subcarriers by preventing ISI, i.e. the cyclic prefix provides a time-delay buffer between OFDM symbols seen at the receiver, and must have a duration equal to or greater than the delay spread of the channel. The addition of the CP to the OFDM symbol can be modeled as: 


$$
\tilde{X}=[x[N-p], x[N-p+1], \cdots, x[N-1], x[0], x[1], \cdots, x[N-1]]
$$

where the length of $\mathrm{p}$ is determined by the number of of multipath parameters as follows:

$$
p \geq L-1
$$

where $L$ is the number of multipath or the length of channel. The reason for Equation (29) is to capture all information in the signal.

For simplicity, let $L=3, p=2$; the received output equation is:

$$
\begin{aligned}
& y(m)=\sum_{l=0}^{L-1} h(l) \tilde{x}(m-l) \\
& m=p, p+1, \cdots, N+p-1
\end{aligned}
$$

Thus, the received output equation is given as:

$$
y=\left[h_{0} h_{1} h_{2}\right] *\left[x_{2} x_{3} x_{0} x_{1} x_{2} x_{3}\right]+w
$$

where $w$ is the noise and $\begin{array}{llll}x_{2} & x_{3}\end{array}$ at the beginning of the symbols is the guardband that is affected by the ISI. Thus, the received output based on the convolution of $x$ and $h$ where $x$ includes the cyclic prefix is given by:

$$
\begin{aligned}
& y(0)=h_{0} x_{2}+w \\
& y(1)=h_{0} x_{3}+h_{1} x_{2}+w \\
& y(2)=h_{0} x_{0}+h_{1} x 3+h_{2} x_{2}+w \\
& y(3)=h_{0} x_{1}+h_{1} x_{0}+h_{2} x_{3}+w \\
& y(4)=h_{0} x_{2}+h_{1} x_{1}+h_{2} x_{0}+w \\
& y(5)=h_{0} x_{3}+h_{3} x_{1}+h_{2} x_{0}+w
\end{aligned}
$$

The received outputs $y(2)$ through $y(5)$ include the actual information and thus processed in order to retrieve the original signal. However, $y(0)$ and $y(1)$ are ignored because they are guard-band for the cyclic prefix. The following equations are the channel impulse response and the corresponding received signal $y$.

$$
\begin{gathered}
h=\left[h_{0}, h_{1}, \cdots, h_{L-1}, 0, \cdots, 0\right] \\
y=[y[p], y[p+1], \cdots, y[N+p-1]]
\end{gathered}
$$

where $y[p, p+1, \cdots, N+p-1]$ is the desired signal, i.e. without the prefix cyclic.

Using Equation (26), the output at the receiver is given by:

$$
y=\tilde{h} F_{N}^{*} X+w
$$

The $h$ matrix which is a result of the circular convolution is expressed as:

$$
\tilde{h}=\left[\begin{array}{cccccccc}
h_{0} & 0 & \cdots & 0 & h_{L-1} & h_{L-2} & \cdots & h_{1} \\
h_{1} & h_{0} & 0 & \cdots & 0 & h_{L-1} & \cdots & h_{2} \\
0 & \cdots & 0 & h_{L-1} & h_{L-2} & \cdots & h_{1} & h_{0}
\end{array}\right]
$$

In this case, we consider the channel under fast fading i.e. the impulse response is changing. Next step is to convert the channel from time domain to the 
the frequency domain using Fast Fourier Transform (FFT) as shown in Equation (24). The mathematical representation using Equation (35) is given by the following:

$$
Y=F_{N} y=F_{N} \tilde{h} F_{N}^{*} X+F_{N} w
$$

We know that that channel in the frequency domain is:

$$
H=\frac{Y}{X}
$$

Thus,

$$
H=\frac{Y}{X}=F_{N} \tilde{h} F_{N}^{*} X
$$

which turns out to be a diagonal matrix as shown below:

$$
F_{N} \tilde{h} F_{N}^{*}=\left[\begin{array}{cccc}
H[0] & 0 & \cdots & 0 \\
0 & H[1] & & \vdots \\
\vdots & & \ddots & \\
0 & \cdots & & H[N-1]
\end{array}\right]
$$

When compare the matrix in time domain, matrix 36 to the matrix in frequency domain, matrix 40 , we can see matrix 40 is reduced in complexity and thus the equalization would much easier since you only have one parameter to deal with for each tap. After we find the channel in frequency domain as shown in matrix 40, we choose an equalization method to equalize the signal. MMSE equalizer in frequency domains proves that it works very well in a fast selective environment. The mathematical representation of the MMSE receiver is shown below:

$$
\hat{X}_{\text {mmse }}(k)=\frac{H^{*}(k)}{|H(k)|^{2}+\sigma_{n}^{2}} Y(k)
$$

\subsection{Contribution}

This section will discuss the proposed method for enhancing the equalization of the channel. More specifically, the enhanced equalization method we are proposing is a combination of a multi-stage time and frequency domain equalizer with a feed-forward loop. Inserting a pilot data at the transmitter and using said pilot data to estimate the channel in time domain. The addition of the feed-forward is utilized to capture a part of the received signal, then extracting the channel information while the signal is being delayed until the information is obtained. The estimated channel is forwarded to the MMSE equalizer. The first stage of the muti-stage equalizer is using pilot data as a measurement data in the frequency domain. The second stage is to convert the measurement data to the time domain. In the third stage, we estimate the channel using the pilot data i.e. the measurement data, i.e. input to the Kalman filter. Taking under consideration that in OFDM system the channel coefficients for each tap, in time domain for- consecutive blocks are not totally independent, but they are correlated; thus such correlation is utilized for time domain channel estimation. Using the 
KALMAN filter, we can extract this information from previous blocks and use them for the newly arrived one, and perform a better channel estimation. The fourth stage, we use the output of the Kalman filter as a feed-forward to the frequency equalizer for each subcarrier in which the data is converted from time domain to frequency domain. Adding efficiency to the channel by dynamically selecting the number of previous states based on the doppler and the autocorrelation of the channel. Furthermore, in order to evaluate the performance of the proposed method, a comparison is conducted with the following methods:

1) A traditional channel equalization using MMSE and IFFT interpolation.

2) A perfect channel equalization where we assume the channel is perfect.

\section{Simulations and Results}

In this section, simulation is performed to compare the performance between LS and MMSE equalizers at certain conditions explained in the following sections. Also, we validate the performance of the proposed multi-stage equalizer, for high frequency networks and high speed mobility, by comparing its performance with other methods.

Figure 5 illustrates a process of the proposed system starting at the transmitter, going through a wireless channel and finally at the receiver. Source data is generated randomly in a bit stream. The bit stream will be converted to a symbol size (bit/symbol). The converted data will be separated into multiple subcarriers by the serial to parallel converter. After that, the data will be modulated utilizing different modulation schemes (QAM, QPSK). A pilot data will be inserted into

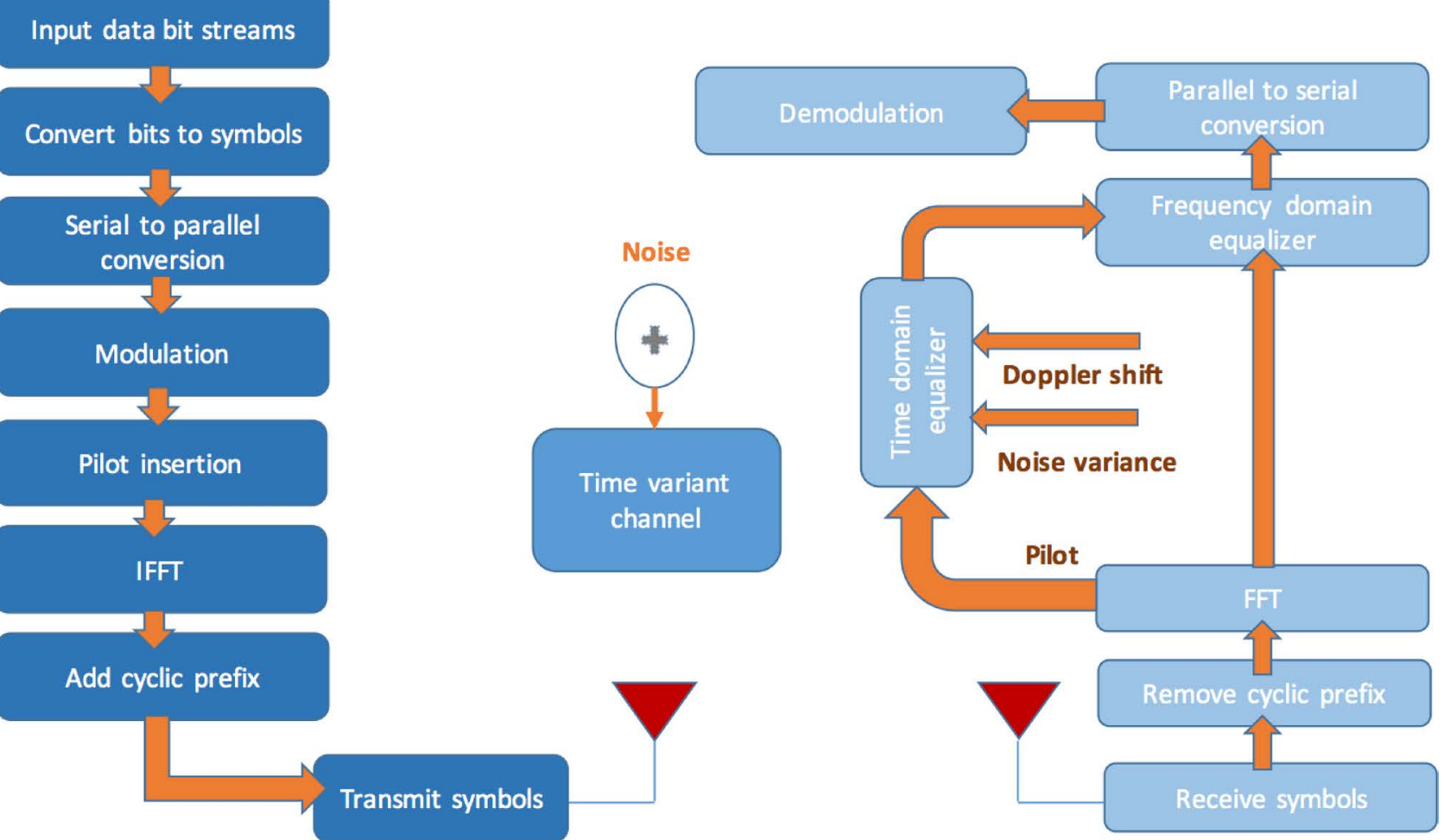

Figure 5. OFDM system process. 
the subcarriers. Prior to transmitting the data, the modulated subcarriers are transformed to time domain using IFFT and cascaded together along with guard interval (cyclic prefix) inserted in between each other. The communication time variant channel is modeled with a Gaussian noise added. At the receiver, the symbols are received and guard intervals are removed. After that, the data is converted from time domain to frequency domain. The pilot data positions and their values are fed into the time domain equalizer where the equalizer reduces the inter-symbol interference and retrieves the original data. With the feed-forward, the output of the time domain equalizer which is transformed to a frequency domain as the well as the output data which is still in frequency domain are fed to the frequency domain equalizer to refine the output. After that, the data is converted from serial to parallel and then demodulated.

\subsection{LS/MMSE}

In this section, we try to explore different channel scenarios, i.e. flat fading channel versus frequency selective channel. Also, we try different types of equalizers, i.e. LS and MMSE. More specifically, we will demonstrate three scenarios with different equalizers techniques, different fading channels, and under certain conditions.

The first scenario is to model a flat fading channel with LS equalizer and analyze the output of said equalizer. The conditions of this scenario are illustrated in Table 1.

The first scenario demonstrates a flat fading channel as shown in Figure 6; wherein said figure shows the flat channel spectrum. We notice in Figure 6 that the spectrum does not show and deep fades. Furthermore, Figure 7 shows the corresponding impulse response for the flat channel with 3 taps, i.e. 3 multi-paths. We notice, as shown in said figure that the impulse at index 2 , is very low relative to the first impulse at index 1 , and impulse at index 3 is very low relative to the one at index 2; wherein the index represents the number of paths. This phenomenon indicates that the interference between adjacent signals is very low, since the adjacent signal is weak and thus less chance of interference.

Figure 8 illustrates the output of the signals with no noise injected into the channel. As shown in said figure, the output after the equalization denoted by det is varying along with the received signal, which is the output signal before equalization denoted by rxsignal, as well as the transmitted signal denoted by signal. Furthermore, Figure 9 shows the constellation shows that the transmitted

Table 1. Flat fading channel conditions.

\begin{tabular}{cc}
\hline Sampling time (Ts) & 1 \\
Signal length & $1 \mathrm{e} 3$ \\
Channel length (l) & 3 \\
Signal to Noise Ratio (SNR) & $20 \mathrm{~dB}$ \\
Channel taps & {$\left[\begin{array}{lll}1 & 1 / 10 & 1 / 100\end{array}\right]$} \\
Noise & No \\
\hline
\end{tabular}




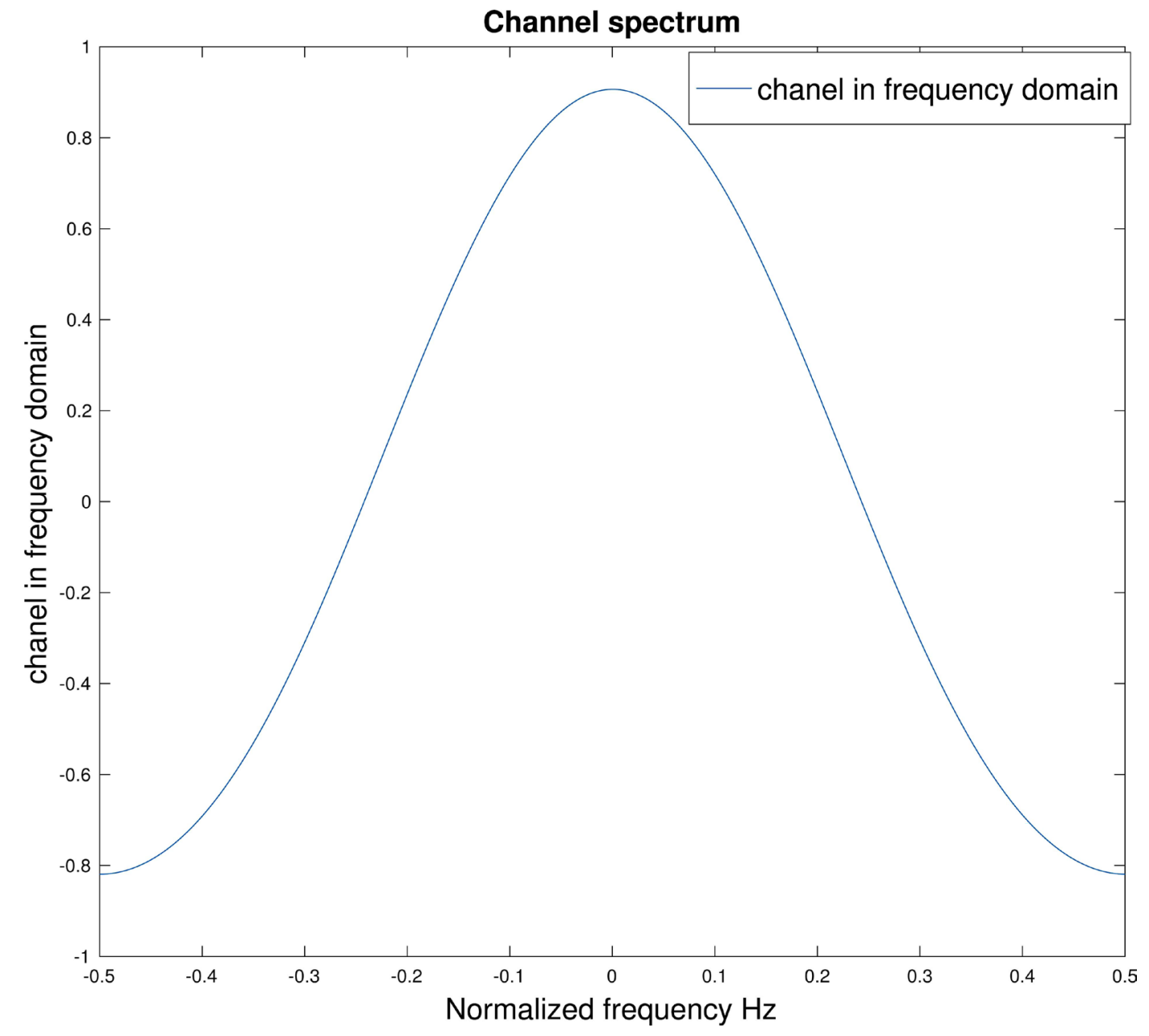

Figure 6. Flat fading.

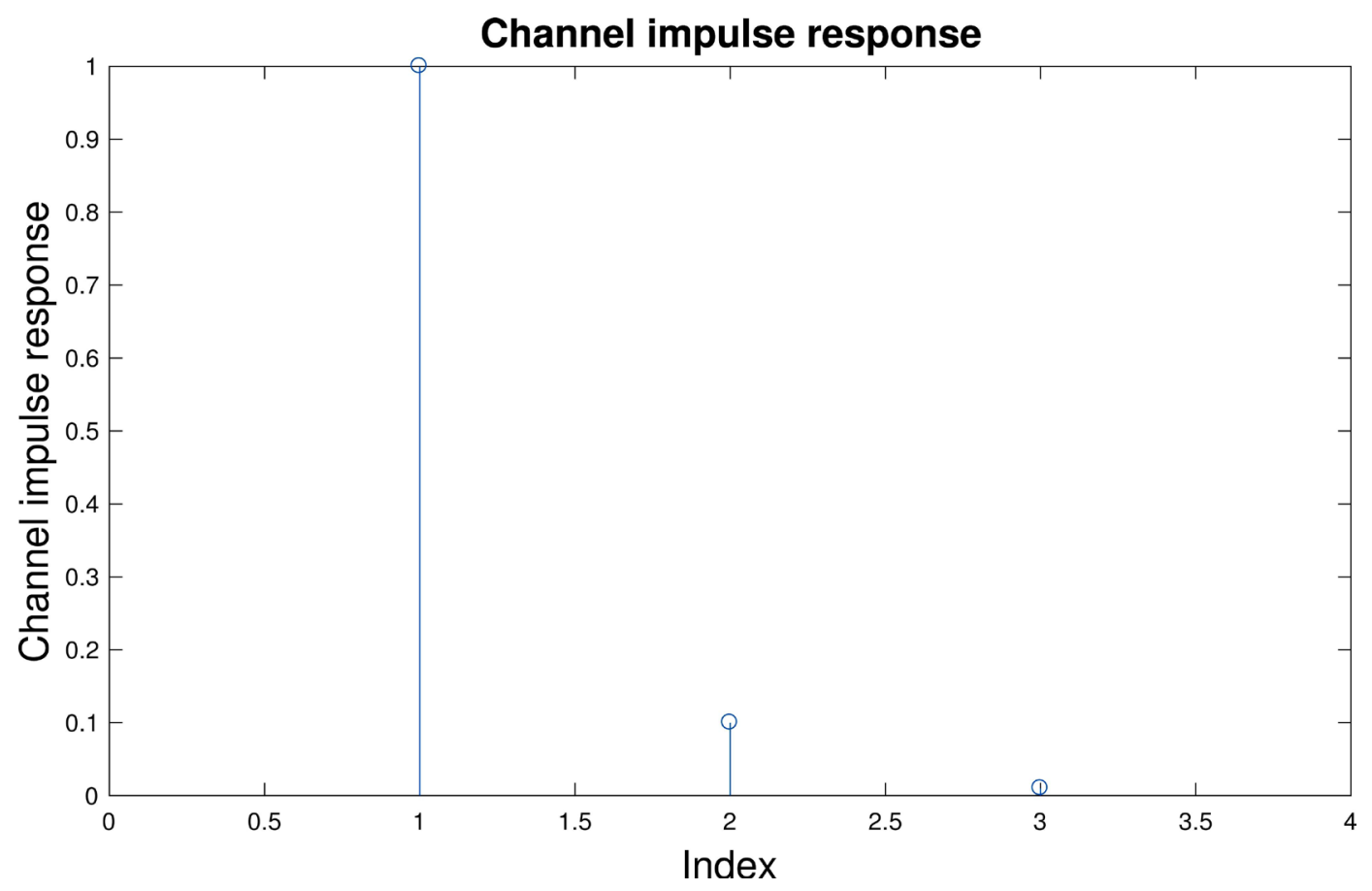

Figure 7. Impulse response for flat fading. 


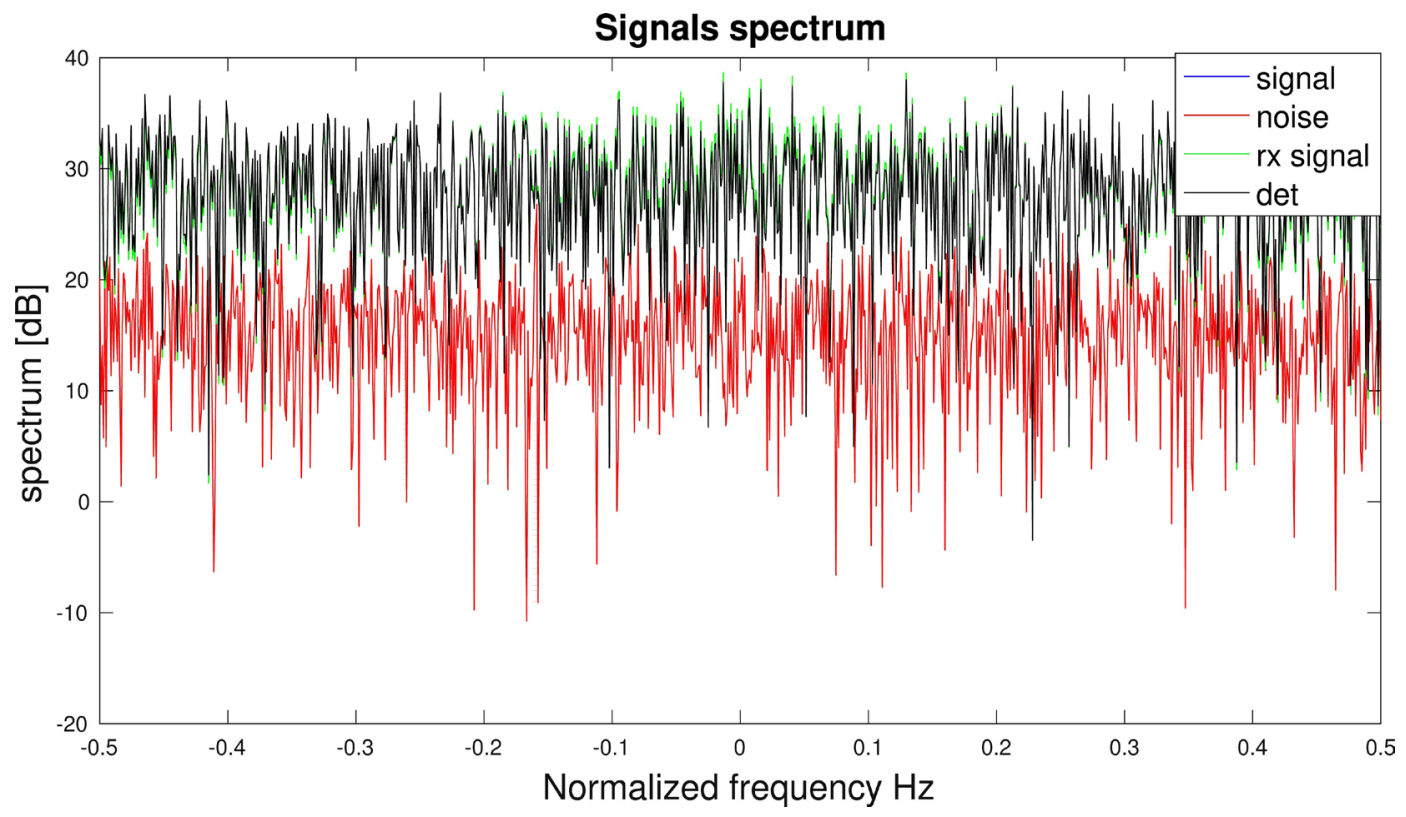

Figure 8. Output signals for LS Equalizer with deep fading channel.

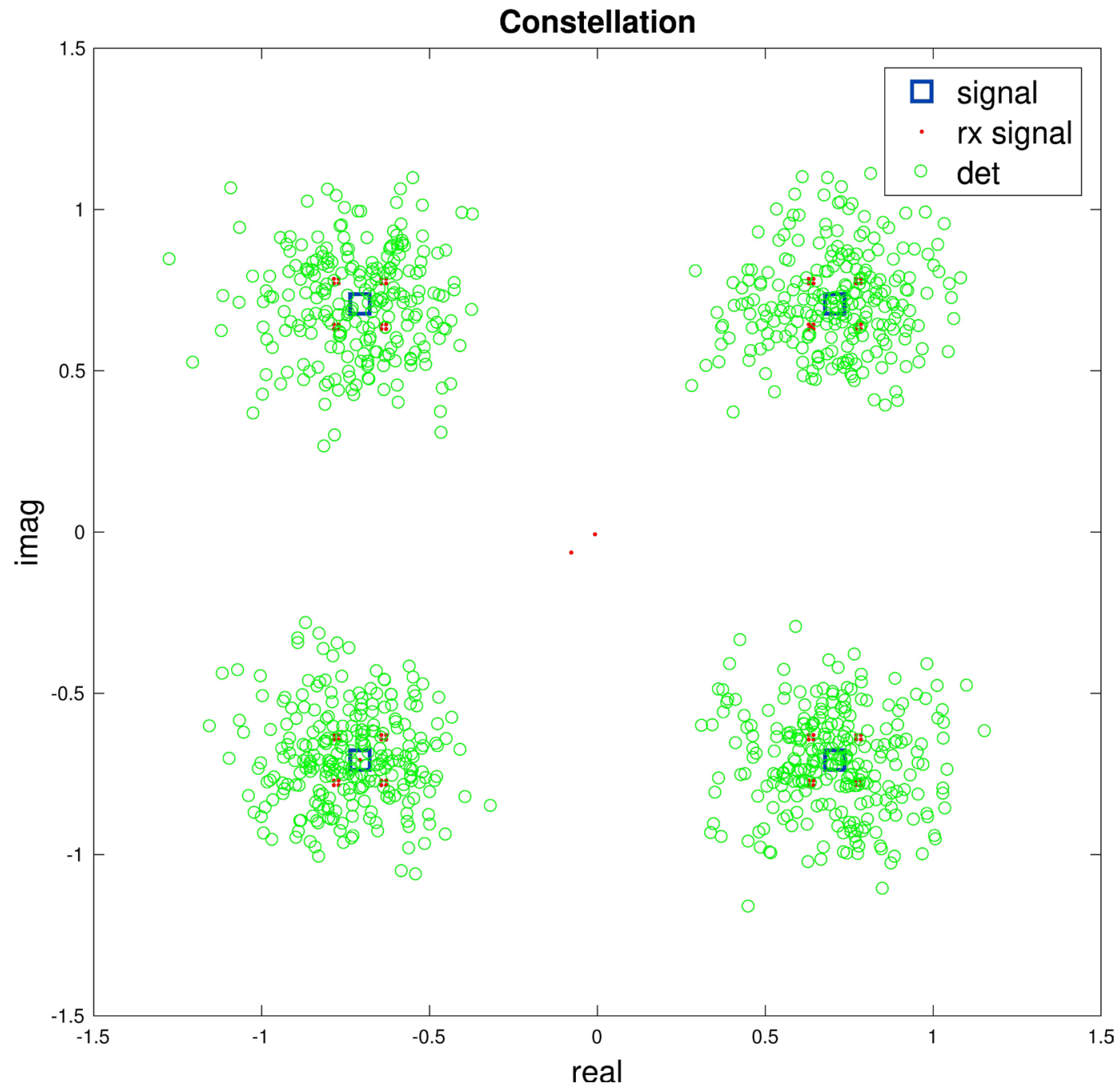

Figure 9. Constellation for flat channel. 
signal denoted by signal, received signal denoted by rxsignal, and the signal after equalization denoted by det are very close to each other.

Subsequently, the second scenario is to model a frequency selective channel fading, i.e. deep fading, with LS equalizer. The conditions are shown in Table 2.

Figure 10 shows a 3 tap channel model with all strong power as a result a deep fading is produced. Furthermore, the channel is injected with Gaussian noise.

As illustrated in Figure 11, a deep fading is introduced at two intervals.

Figure 12 shows the received signal after equalization has a high power at deep fading regions. This means that the noise is higher than the actual signal, i.e. the noise at the deep regions is amplified due to the nature of LS equalizer. Furthermore, Figure 13 shows a constellation of the deep fading channel with noise. As shown in the figure, the constellation is very noisy and as a result we can clearly draw a conclusion that the LS equalizer is not a commendable fit for this type of channel, i.e. for the deep fading channel with noise.

The third scenario is to utilize MMSE Equalizer in place of LS Equalizer with

Table 2. Frequency selective fading channel conditions.

\begin{tabular}{cc}
\hline Sampling time (Ts) & 1 \\
Signal length & $1 \mathrm{e} 3$ \\
Channel length (l) & 3 \\
Signal to Noise Ratio (SNR) & $20 \mathrm{~dB}$ \\
Channel taps & {$\left[\begin{array}{lll}1 & 0.99 & 0.95\end{array}\right]$} \\
Noise & Yes \\
\hline
\end{tabular}

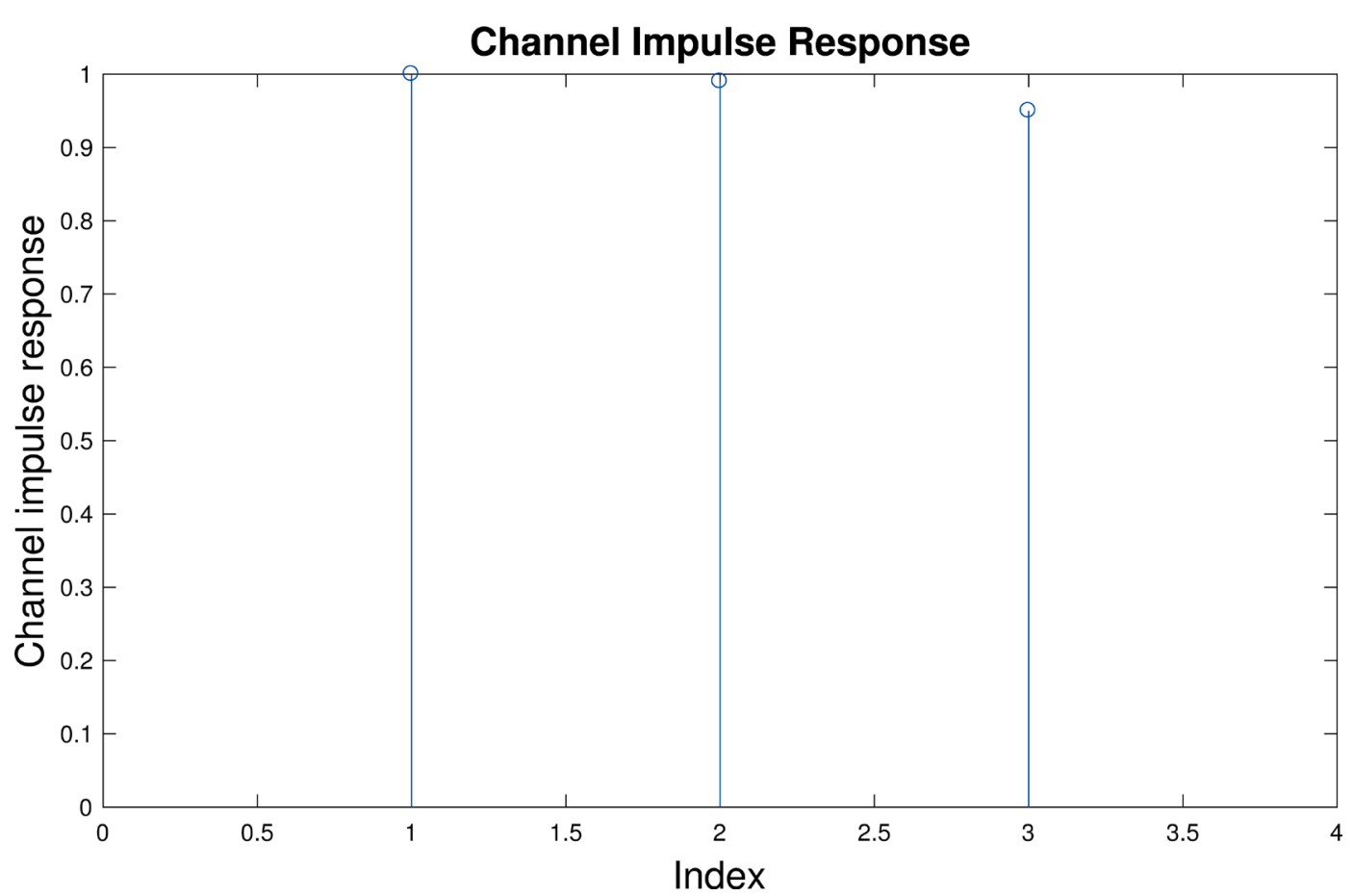

Figure 10. Impulse Response for LS Equalizer with deep fading channel. 


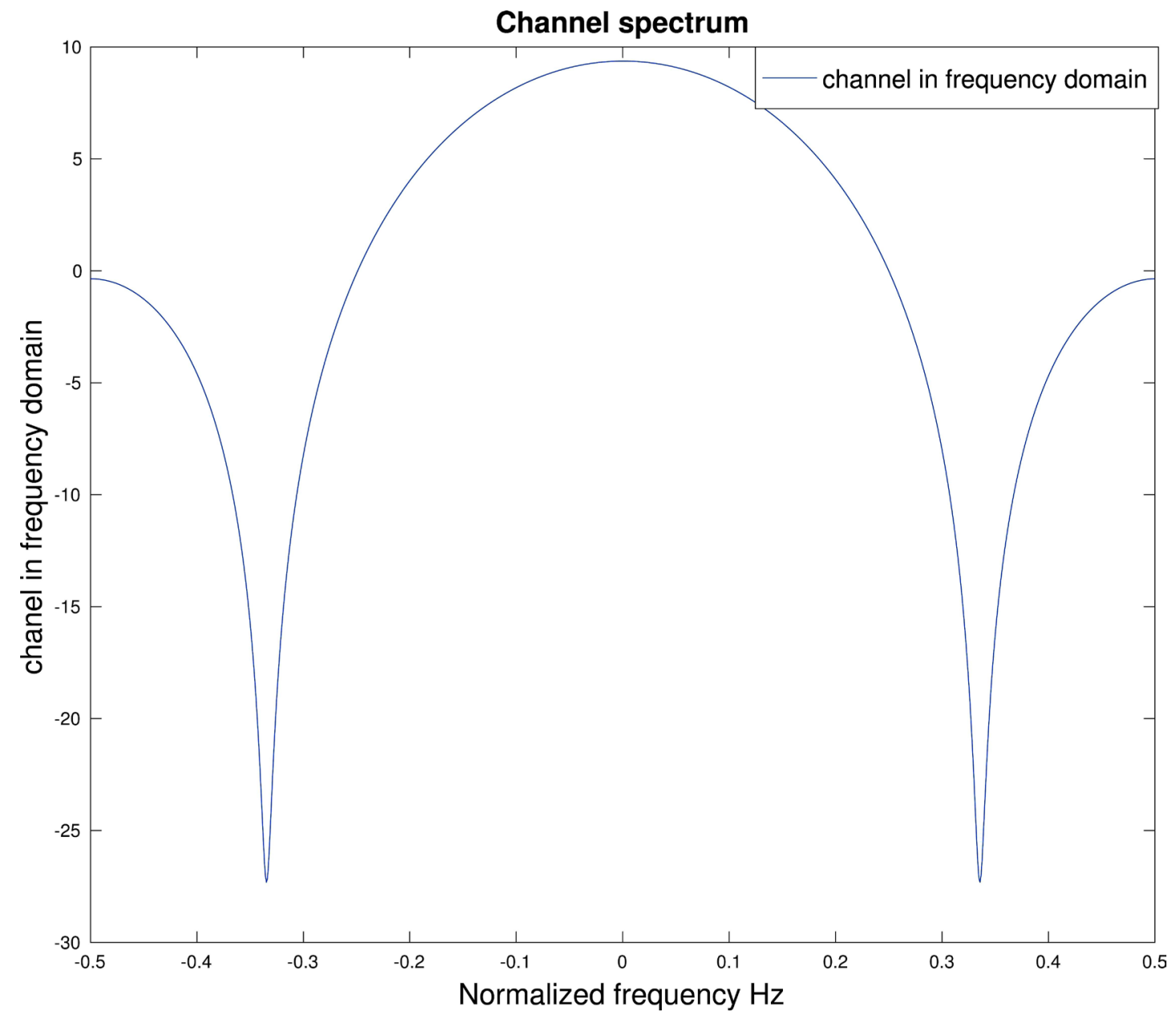

Figure 11. Deep fading.

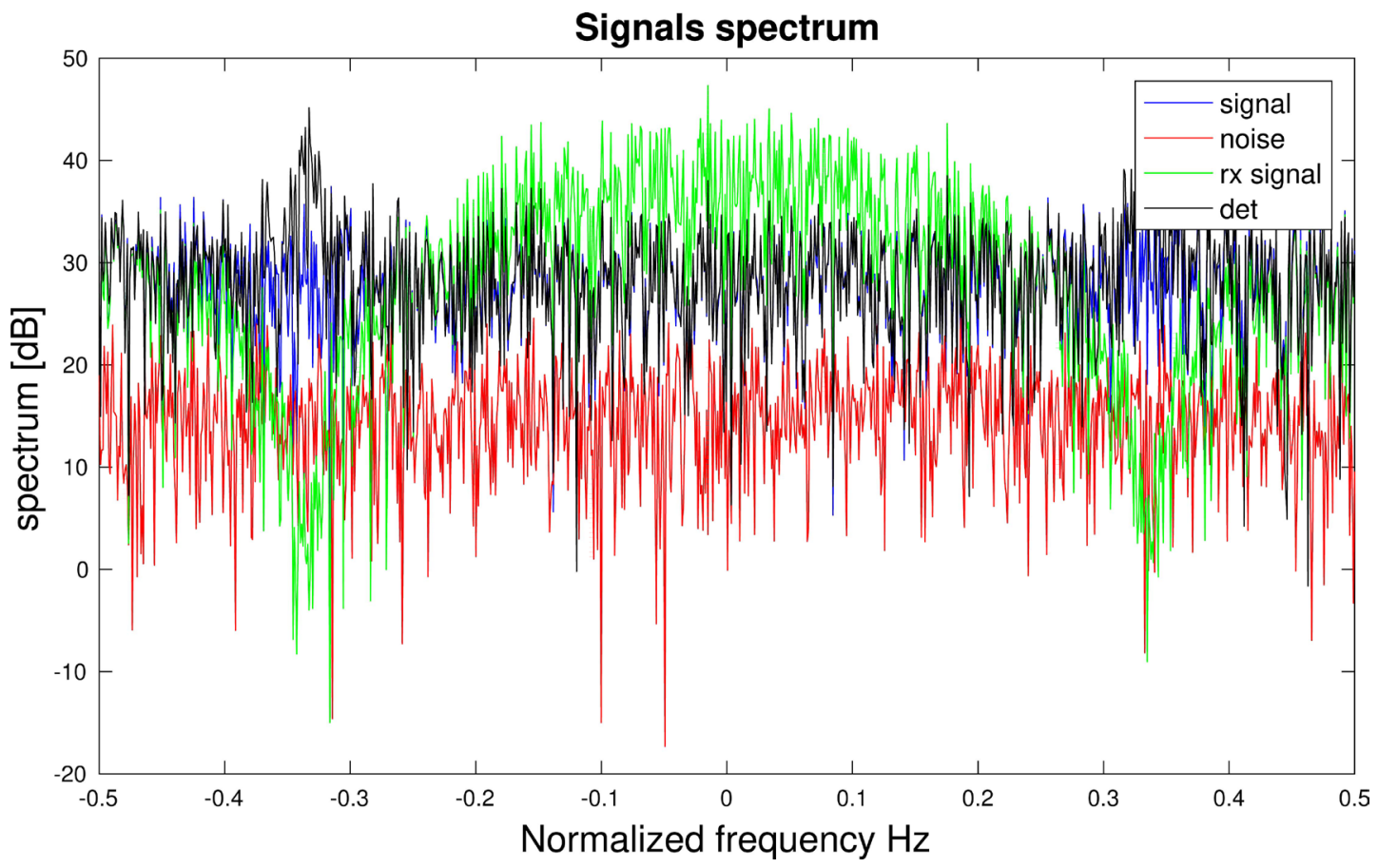

Figure 12. Output signals for LS Equalizer with deep fading channel. 


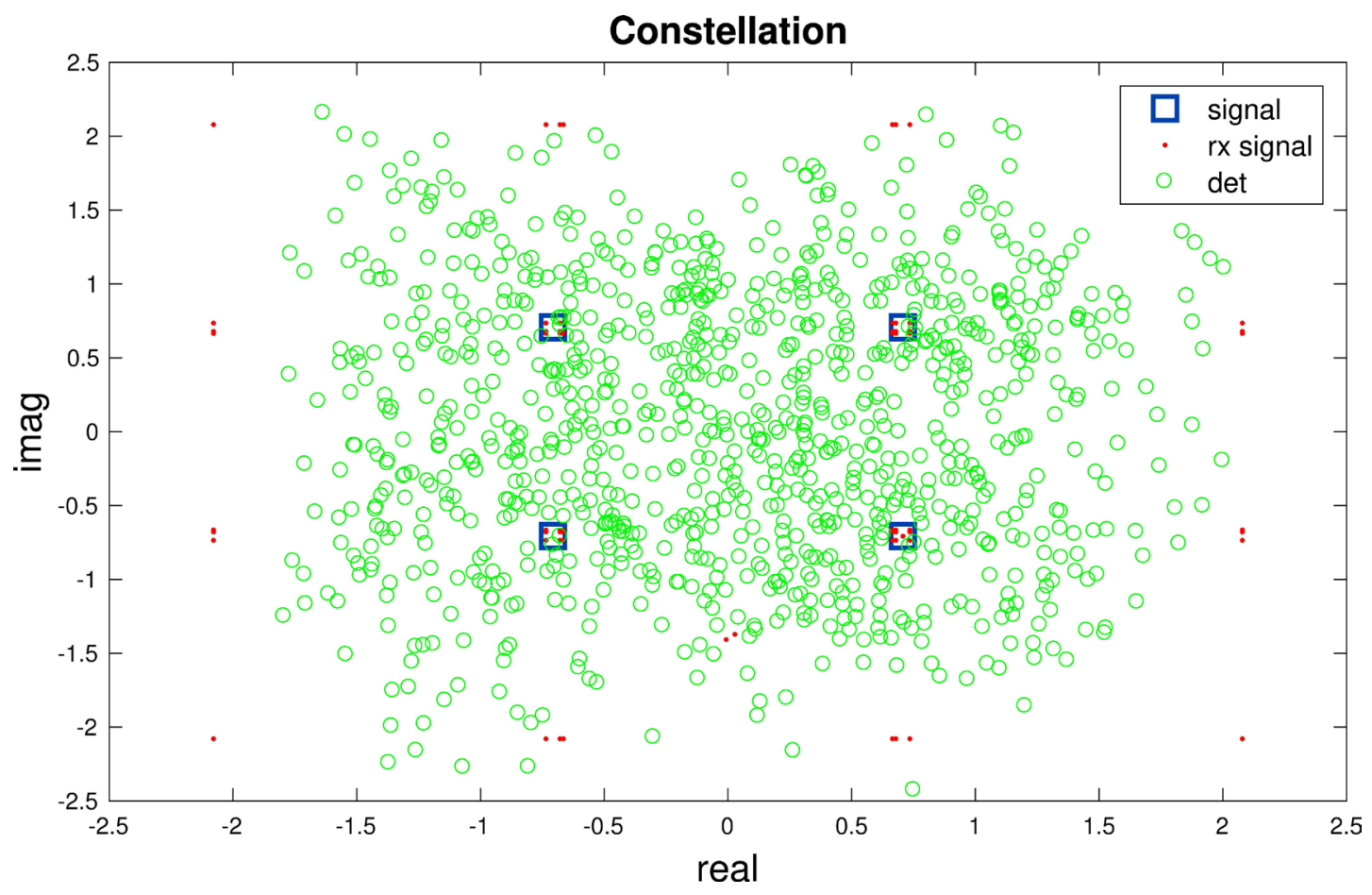

Figure 13. Constellation for the LS Equalizer with deep fading channel.

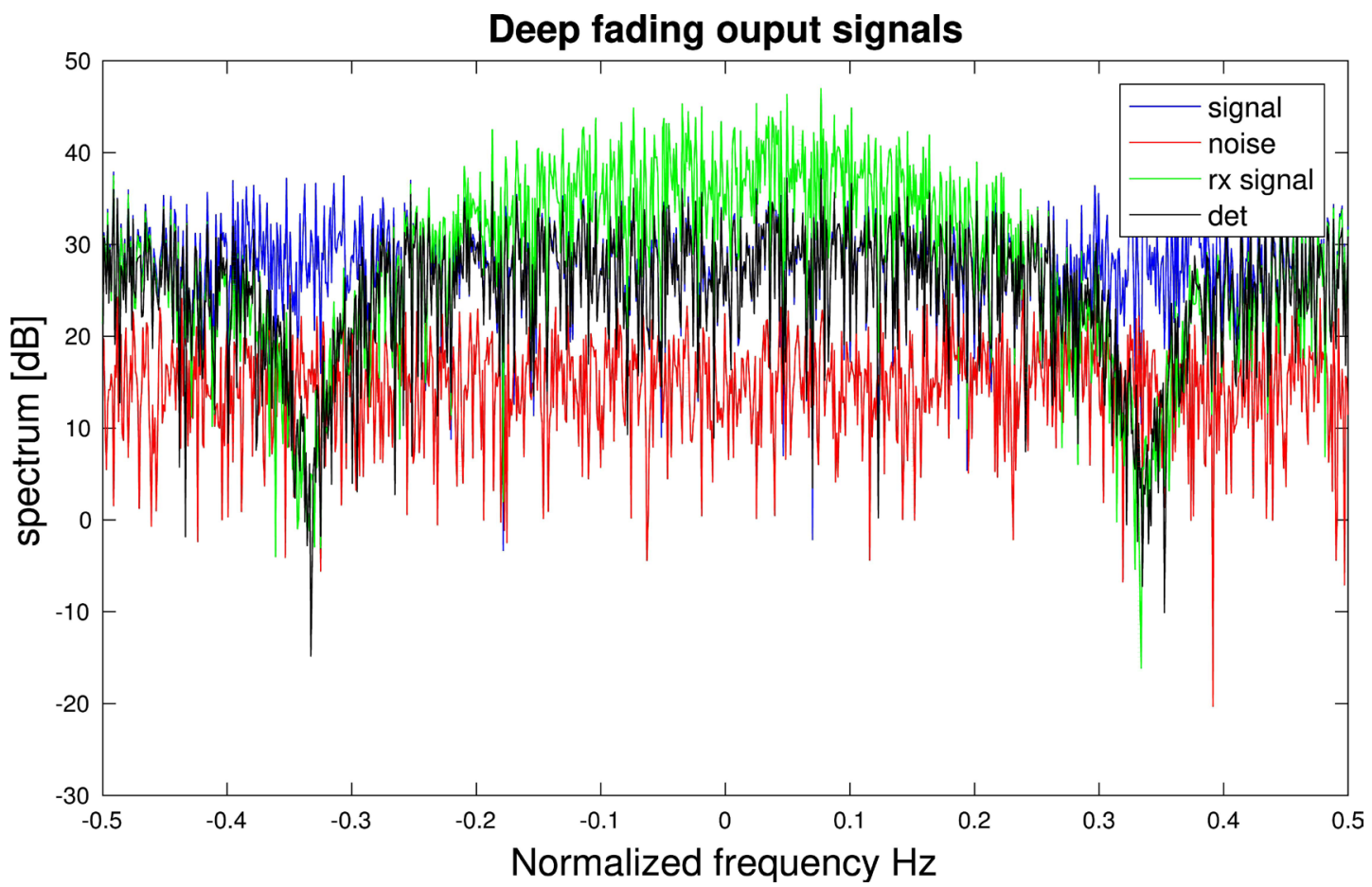

Figure 14. Signal outputs for the MMSE Equalizer with deep fading channel.

conditions illustrated in Table 2. Figure 14 shows that MMSE is a much better Equalizer than LS Equalizer. Thus, the noise is suppressed at deep fading regions. Furthermore, Figure 15 shows the constellation of the signals, wherein the received signal has less noise relative to the LS Equalizer constellation. Thus, both figures demonstrate that MMSE is much better than LS equalizer. 


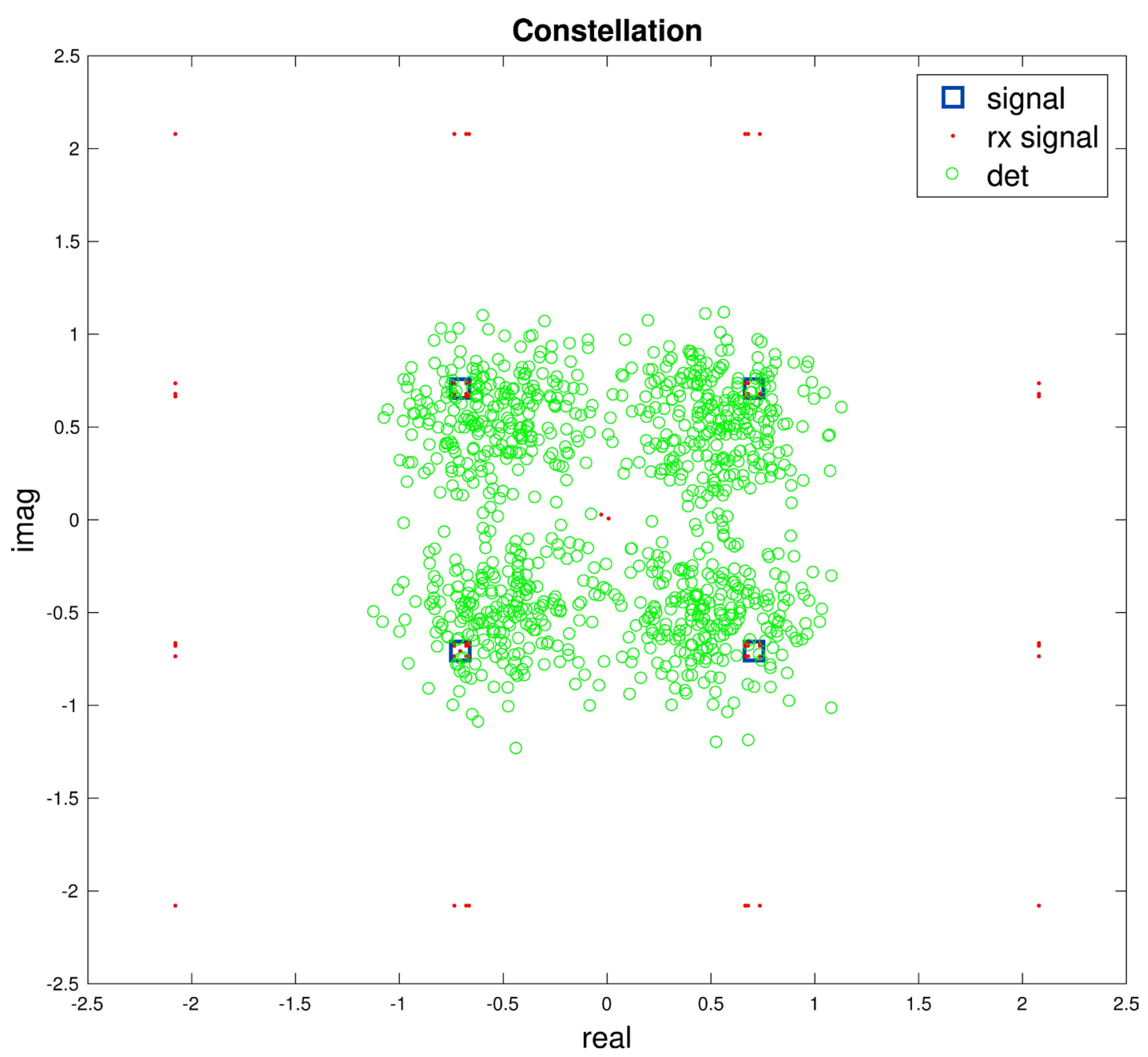

Figure 15. Constellation for the MMSE Equalizer with deep fading channel.

\subsection{Multi-Stage Equalizer}

In this section, we validate the performance of the proposed multi-stage equalizer, for high frequency networks and high speed mobility, by comparing its performance with other methods. The channel model used in this paper is Rayleigh channel model with additive white Gaussian noise. The parameters in Table 3 represent OFDM parameters as well as the conditions of the surrounding environment. The total number of subcarriers is 256 with 8 of them used for pilot transmission and 248 used for data transmission, and quadrature amplitude modulation (QAM) was employed.

Figure 16 depicts the signal frequency spectra using the Spectrum Analyzer block in simulink; wherein the spectrum analyzer measures the magnitude of the signal versus the frequency within the full frequency range. As shown in said figure, the bandwidth of the signal is around $2 \mathrm{MHz}$. Furthermore, Figure 17 shows the frequency response of the channel; wherein the channel shows certain distortions known as "deep fading". As shown said figure, the deep fading is represented by the signal drops at certain intervals. This is expected since the channel we are dealing with is such a dynamic channel with multi-paths. Fur- 
Table 3. Frequency selective fading channel conditions.

\begin{tabular}{cc}
\hline Parameters & Values \\
\hline Bandwidth & $4 \mathrm{MHz}$ \\
Total subcarriers & 256 \\
Subcarrier spacing & $12 \mathrm{KHz}$ \\
Modulation & QAM \\
Doppler & {$[36200] \mathrm{Hz}$} \\
CP length & $5 \mu \mathrm{sec}$ \\
SNR & {$[5: 3: 40] \mathrm{dB}$} \\
Number of pilots & 8 \\
& \\
\hline
\end{tabular}

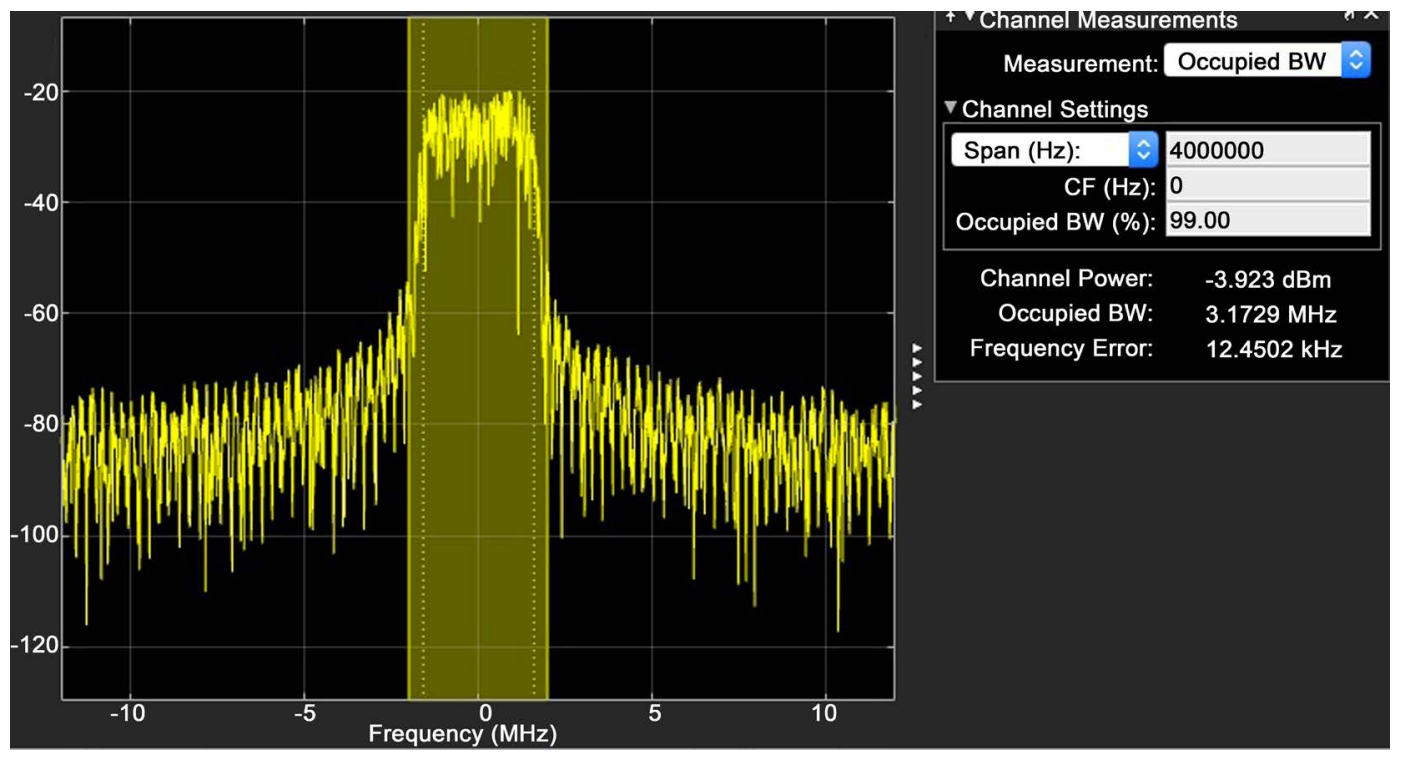

Figure 16. Signal frequency spectrum.

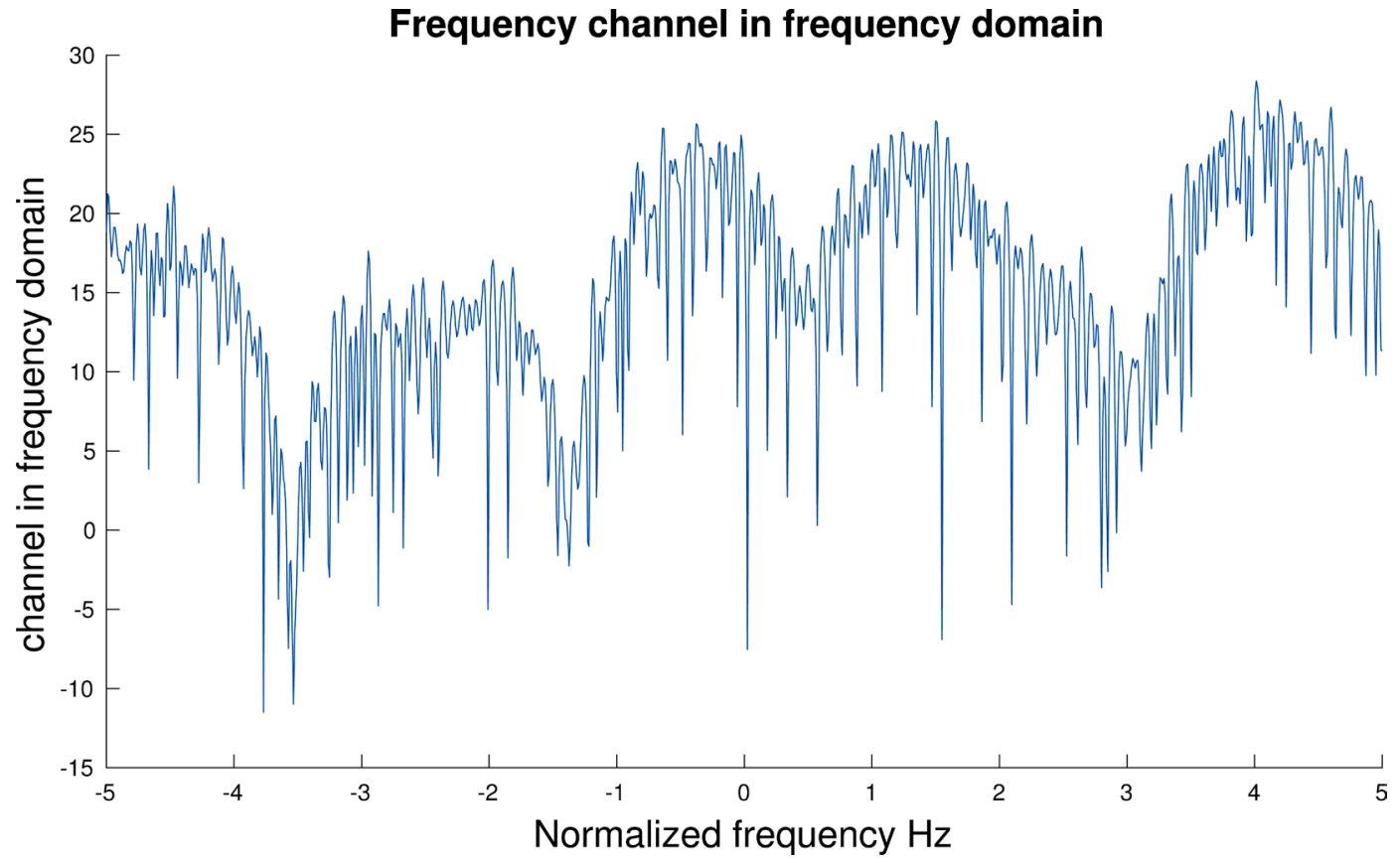

Figure 17. Frequency response of the channel. 


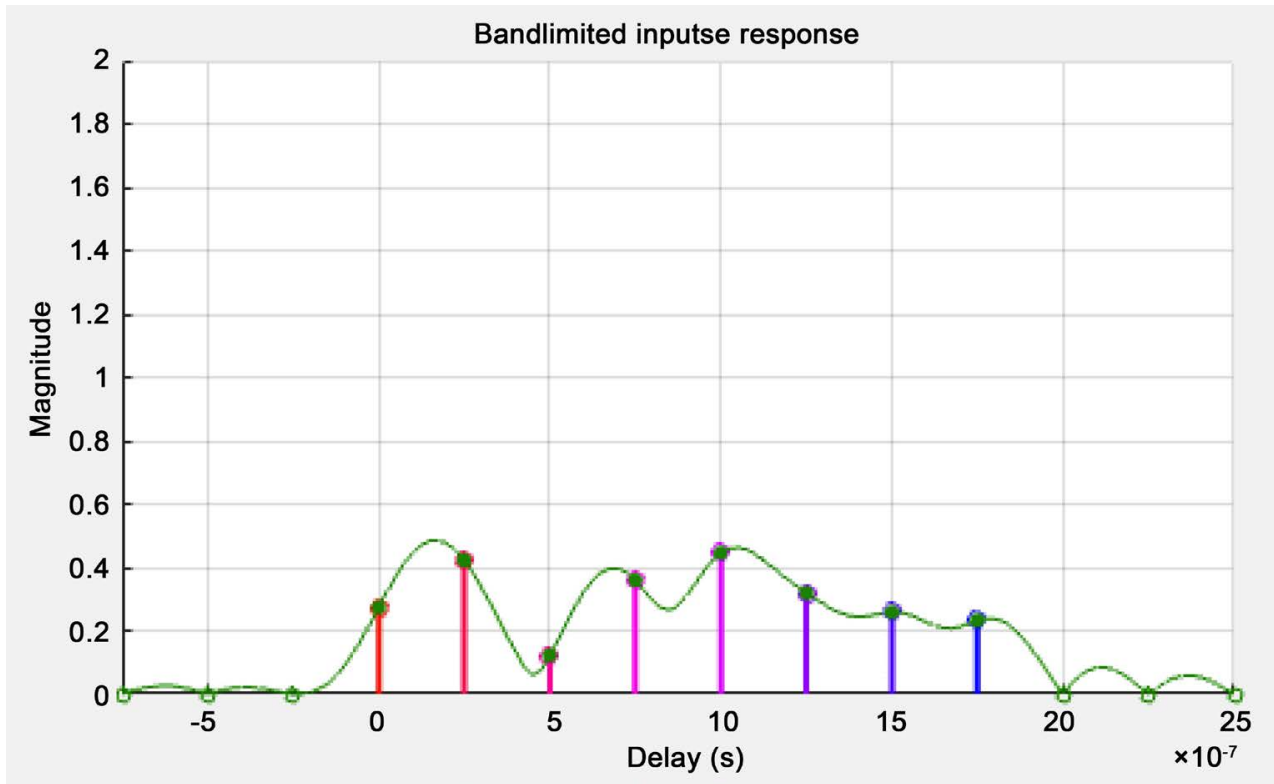

Figure 18. Channel impulse response.

thermore, the speed and doppler components are considered to have major contribution to the fading channel.

Figure 18 shows the impulse response of the channels. In Figure 18, a representation of a time varying propagation behavior of multipath components is illustrated. The multipath components exhibit a delay of each tap as well as fluctuation of magnitude.

The simulation is performed at three different speeds, different noise levels with SNR ranging from $5 \mathrm{~dB}$ to $40 \mathrm{~dB}$ in order to have a wide spectrum of error rate. Figure 19 and Figure 20 depict the BER of the multi-stage equalizer along with the theoretical BER, and traditional method. As for a performance benchmark, the BER performance of a perfect channel estimation algorithm is depicted. As expected, the improvement of BER in the proposed method, can be seen clearly, gives the best performance, i.e. achieving a smaller error. The Mean square (MSE) of the proposed method is in the range of -20 and $-10 \mathrm{~dB}$ and that of the traditional method is -14 and $-5 \mathrm{~dB}$.

Figure 21 shows an instantaneous channel performance of based on the multi-stage equalizer algorithm over OFDM subcarriers along with the benchmark algorithms, perfect channel estimation, pilot symbols, and the traditional estimation algorithm. It is evident that the proposed method leads to the closest trajectory to the perfect channel and the pilot symbols. The multi-stage equalizer-based algorithm proposed provides better performances in terms of MSE. Nevertheless, the estimation error of our algorithm is small enough to guarantee better bit error rates (BERs) comparing to the traditional algorithm.

While the performance of the proposed method, Multi-stage Equalizer, is being analyzed and explored, said method is performed with respect to two major parameters, Doppler, and number or previous states. In this paper, we emphasize on the relationship between these two parameters in order to improve the 


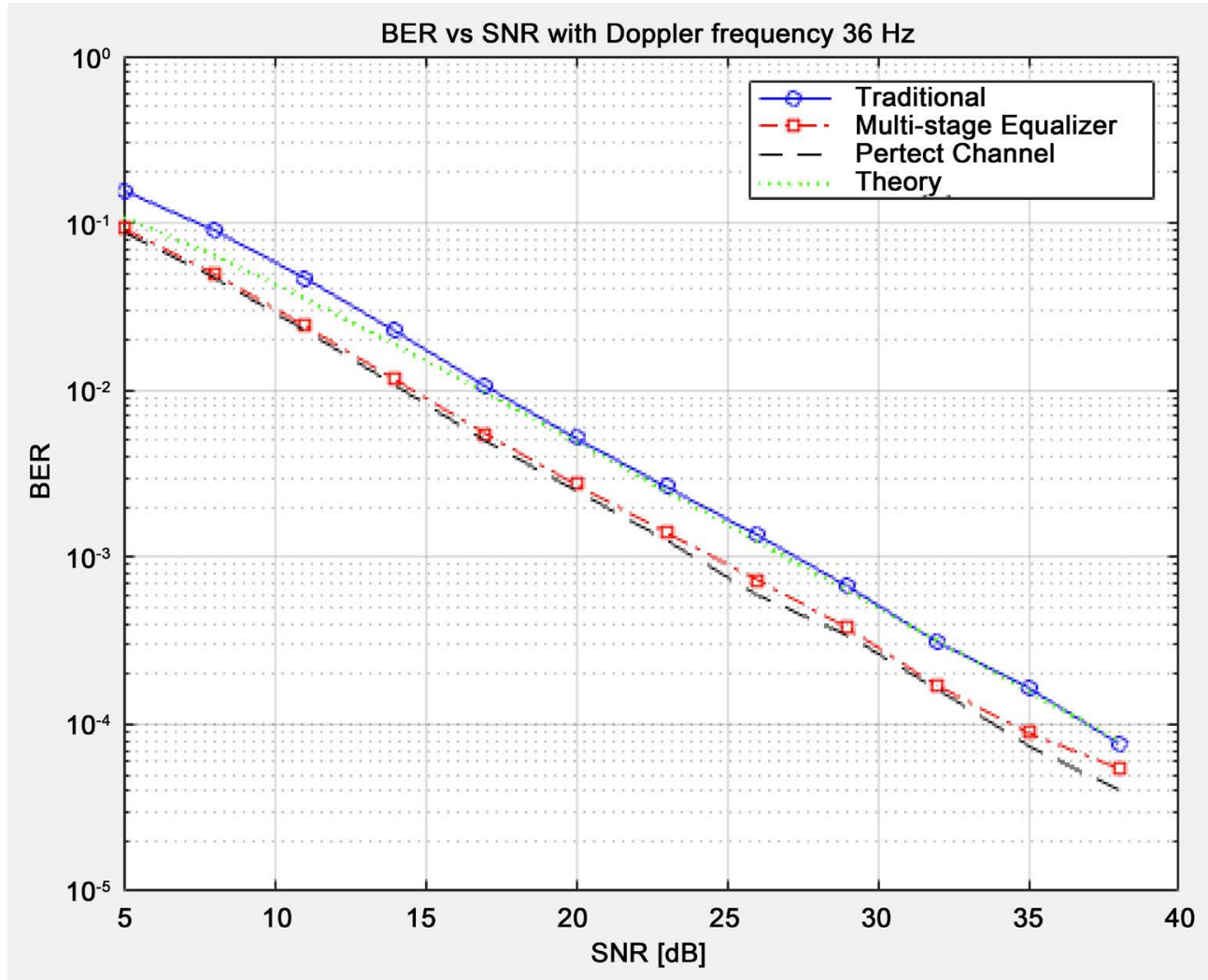

Figure 19. A comparison of BER of the traditional method versus the proposed method at $36 \mathrm{~Hz}$.

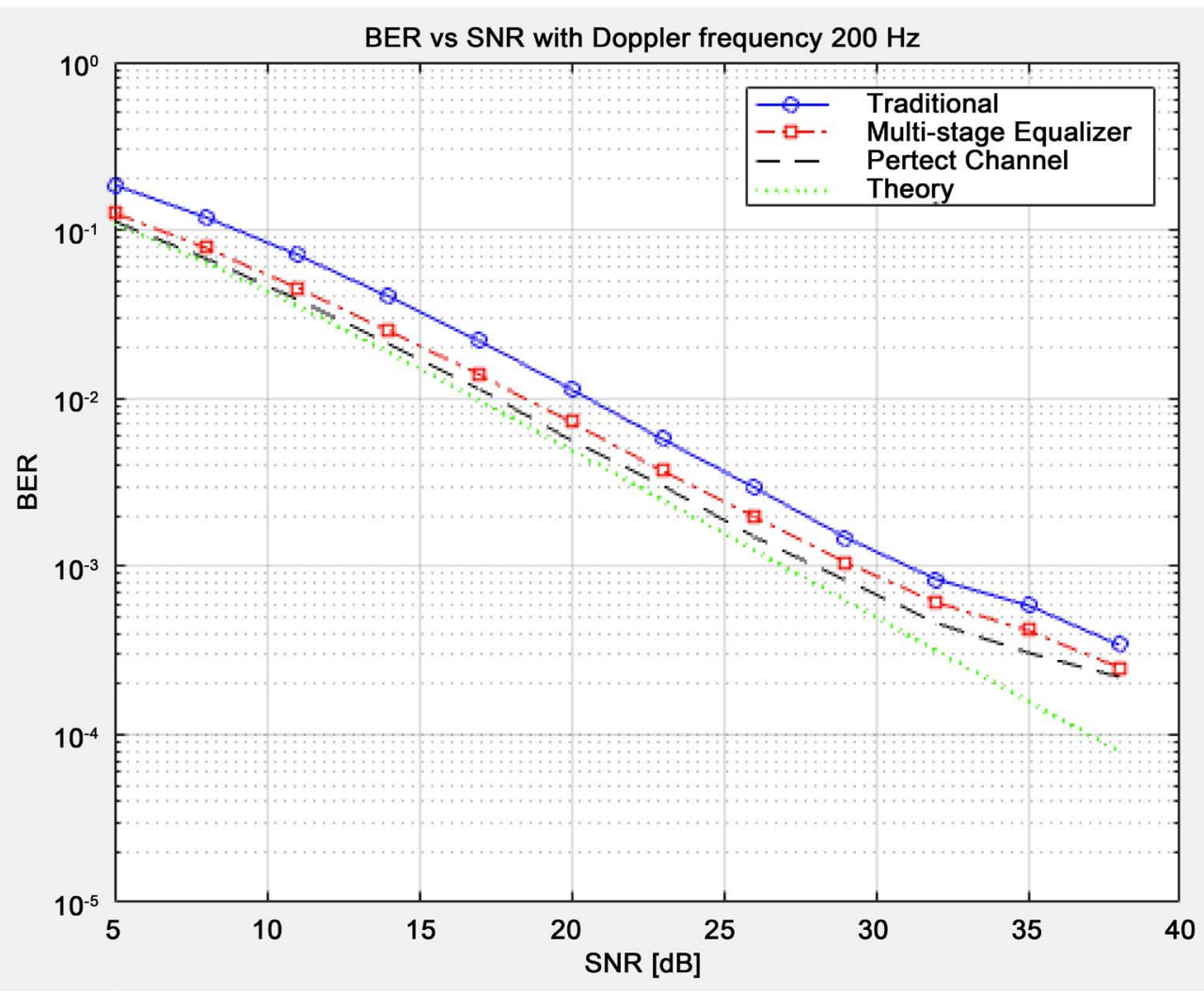

Figure 20. A comparison of BER of the traditional method versus Multi-stage Equalizer at 100 $\mathrm{Km} / \mathrm{hr}$. 
Frequency domain channel estimation (last $\mathrm{T}_{\mathrm{x}}$ symbol)

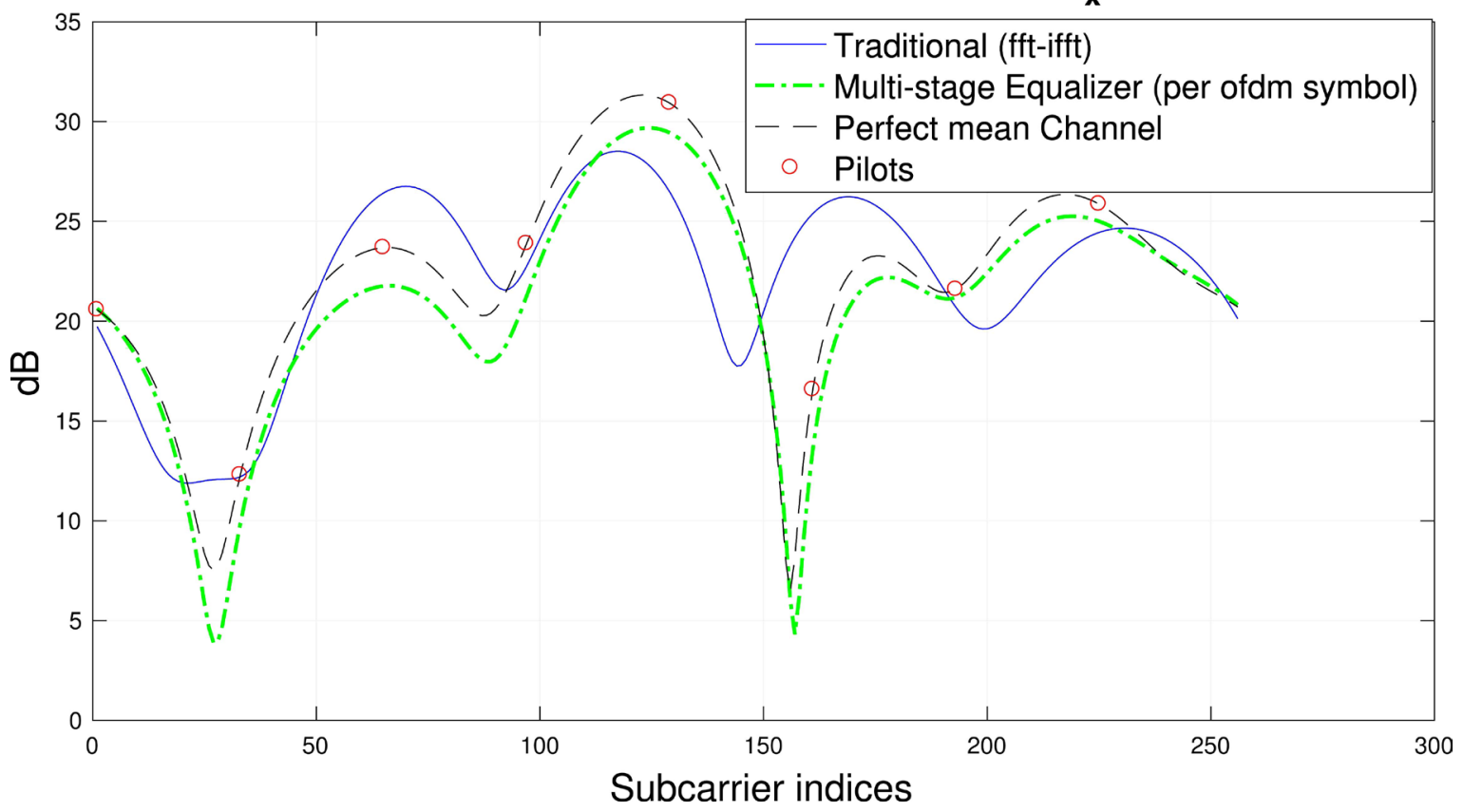

Figure 21. Channel estimation performance comparision.

channel equalization. Figure 22 depicts a $2 \mathrm{D}$ graph where a plot of the autocorrelation with respect to Doppler and number of previous samples is illustrated. The plot clearly shows at low Doppler, the higher number of previous samples results into a higher correlation. In other words, increasing the number of samples would indeed result in a more accurate estimation. These observations are critical, for example, in order to improve the channel equalization while said channel is dynamically changing, i.e. Doppler is not being constant, there must be an effective mechanism to adaptively and dynamically select number of previous samples. However, as the Doppler increases, the correlation between the previous samples and the Doppler decreases. That translates to the fact that after certain Doppler range, increasing the number of previous samples does not help the estimation. Thus, by the relationship shown in Figure 22, one can select relatively minimum number of previous samples while maintaining a high accuracy.

Figure 23 shows a correlation between BER, Doppler, and number of previous samples. To prove what was shown in Figure 22, at a high frequency range, and high number of previous samples, BER does not really change. Basically, the correlation is low at high Doppler, therefore increasing the number of previous samples does not improve the performance.

\section{Conclusion}

Channel Equalization has been proved throughout the years to improve the channels performance under certain conditions. Channel equalization has been successfully utilized in many applications such as video, image, voice etc. However, as a result of certain critical conditions such as higher frequency and speed, 


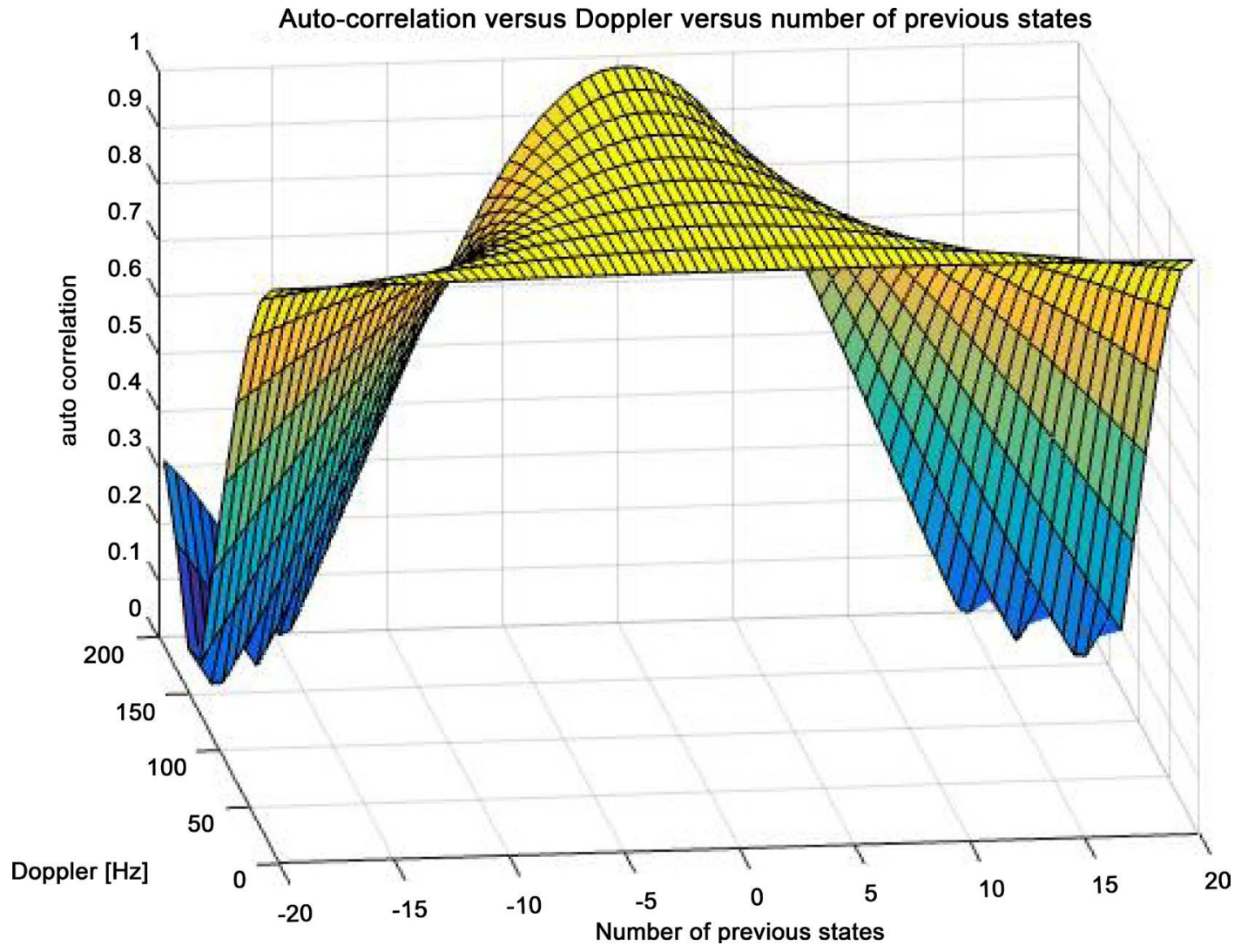

Figure 22. AutoCorrelation versus Doppler and Number of previous samples.

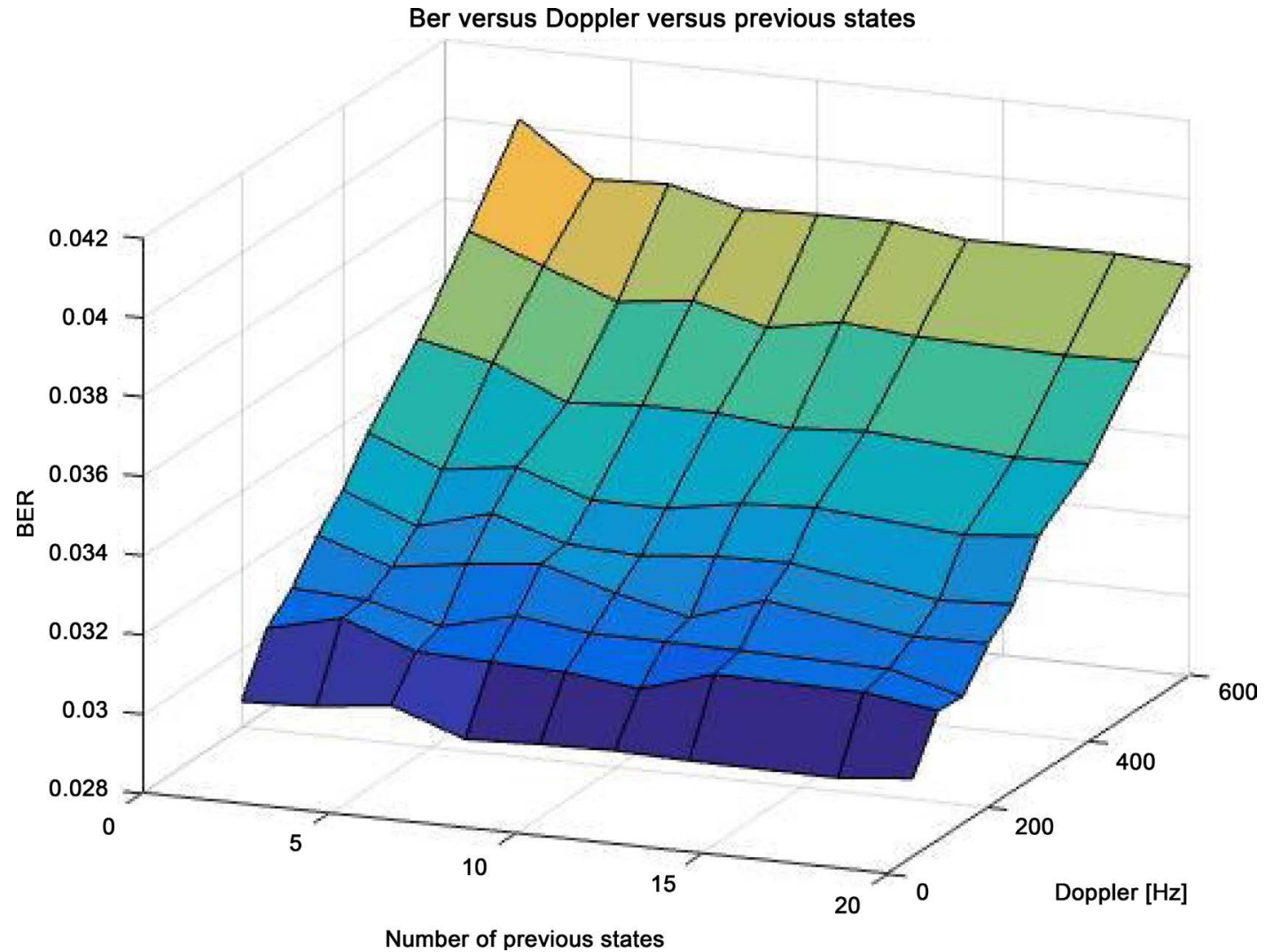

Figure 23. AutoCorrelation versus Doppler and Number of previous states. 
the complexity of the system tends to increase and the performance of the system tends to deteriorate. Many Equalization techniques have been proposed to optimize the performance of the wireless channel in such a dynamic environment, however these techniques still use very complex algorithms. In this paper, we have proposed a unique method for enhancing the accurate equalization of time-variant Rayleigh channels under dynamic changes. Significant performance gains were observed for such selective fading channels when compared to the traditional method. Our theoretical and simulation results demonstrate that to achieve such high accurate performance, knowledge of the previous samples of the channel is required however the number of the previous samples depends on the Doppler range. We also compared both LS and MMSE equalizers under different channel conditions. Simulations showed that MMSE equalizer is better than LS under critical channel. In this paper, we present a low-complexity multi-stage equalization algorithm for an OFDM system, utilized for higher frequency network such as but not limited to fourth generation (4G) communication system. The proposed multi-stage algorithm utilizes guard interval in time domain to eliminate inter-symbol-interference. Furthermore, the approach does not require the use of many pilot data for the equalizer improvement, therefore saving the channel bandwidth. To obtain significant performance improvement over the traditional equalizers, a multi-stage algorithm, time domain equalizer Kalman filter, has been adapted in conjunction with frequency domain equalizer, MMSE. Through simulations, the multi-stage algorithm is shown to be superior to the traditional algorithms.

\section{References}

[1] Baddour, K.E. and Beaulieu, N.C. (2005) Autoregressive Modeling for Fading Channel Simulation. IEEE Transactions on Wireless Communications, 4, 1650-1662. https://doi.org/10.1109/TWC.2005.850327

[2] Scammell, D., Hammoudeh, A. and Sanchez, M.G. (2004) Estimating Channel Performance for Time Invariant Channels. Electronics Letters, 40, 746-747. https://doi.org/10.1049/el:20040508

[3] Mawari, R., Henderson, A., Akbar, M., Durgin, G. and Zohdy, M. (2016) An Improved Characterization of Small Scale Fading Based on 2D Measurements and Modeling of a Moving Receiver in an Indoor Environment. Journal of Signal and Information Processing, 7, 160-174.

[4] Takahashi, Y., Iwanami, Y. and Okamoto, E. (2007) A Time-Domain Block Equalization Scheme on SIMO Frequency Selective Channels. 2007 IEEE Region 10 Conference TENCON, Taipei, 30 October-2 November 2007, 1-4. https://doi.org/10.1109/TENCON.2007.4428938

[5] Cho, Y.-O. (2006) Method and Apparatus for Transmitting/Receiving a Signal in an FFH-OFDM Communication System. US 2006/0268675.

[6] Arun, C.A. and Prakasam, P. (2016) Design of High Speed FFT Algorithm for OFDM Technique. Conference on Emerging Devices and Smart Systems (ICEDSS), Namakkal, 4-5 March 2016, 66-71. https://doi.org/10.1109/ICEDSS.2016.7587780

[7] Alghamdi, A. and Zohdy, M. (2015) Robust Non-Coherent Demodulation Scheme for Bluetooth Voice Transmission Using Linear, Extended, and Unscented Kalman 
Filtering. Journal of Signal and Information Processing. Journal of Signal and Information Processing, 6, 9-27. https://doi.org/10.4236/jsip.2015.61002

[8] Ali, M. and Zohdy, M. (2012) Interactive Kalman Filtering for Differential and Gaussian Frequency Shift Keying Modulation with Application in Bluetooth. Journal of Signal and Information Processing, 3, 63-76.

https://doi.org/10.4236/jsip.2012.31009

[9] Qian, Y., Hang, L. and He, Z. (2004) Iterative Detection for High Rate Single-Carrier Space-Time Block Coded Transmissions over Frequency-Selective Fading Channels. Proceedings of 2004 International Symposium on Intelligent Multimedia, Video and Speech Processing, 20-22 October 2004, 69-72. https://doi.org/10.1109/ISIMP.2004.1434002

[10] Tang, S., Peng, K., Gong, K., Song, J., Pan, C. and Yang, Z. (2008) Novel DecisionAided Channel Estimation for TDS-OFDM Systems. IEEE International Conference on Communications, Beijing, 19-23 May 2008, 946-950.

https://doi.org/10.1109/ICC.2008.185

[11] Yang, F., Wang, J., Wang, J., Song, J. and Yang, Z. (2008) Novel Channel Estimation Method Based on PN Sequence Reconstruction for Chinese DTTB System. IEEE Transactions on Consumer Electronics, 54, 1583-1589. https://doi.org/10.1109/TCE.2008.4711205

[12] Kim, J., Lee, S. and Seo, J. (2006) Synchronization and Channel Estimation in Cyclic Postfix Based OFDM System. IEEE 63rd Vehicular Technology Conference, Melbourne, 7-10 May 2006, 2028-2032.

[13] Bolcskei, H. (2006) MIMO-OFDM Wireless Systems: Basics, Perspectives, and Challenges. IEEE Wireless Communications, 13, 31-37.

https://doi.org/10.1109/MWC.2006.1678163

[14] Tang, S., Peng, K., Gong, K. and Yang, Z. (2008) Channel Estimation for Cyclic Postfixed OFDM. International Conference on Communications, Circuits and Systems, Fujian, 25-27 May 2008, 246-249. https://doi.org/10.1109/ICCCAS.2008.4657769

[15] Liu, G. and Zhang, J. (2007) ITD-DFE Based Channel Estimation and Equalization in TDS-OFDM Receivers. IEEE Transactions on Consumer Electronics, 53, 304309. https://doi.org/10.1109/TCE.2007.381692

[16] Langton, C. (2017) Orthogonal Frequency Division Multiplex (OFDM).

[17] Chen, W. and Zhang, R. (2004) Kalman-Filter Channel Estimator for OFDM Systems in Time and Frequency-Selective Fading Environment. IEEE International Conference on Acoustics, Speech, and Signal Processing, 4, iv-377-iv-380. https://doi.org/10.1109/ICASSP.2004.1326842 
Submit or recommend next manuscript to SCIRP and we will provide best service for you:

Accepting pre-submission inquiries through Email, Facebook, LinkedIn, Twitter, etc. A wide selection of journals (inclusive of 9 subjects, more than 200 journals)

Providing 24-hour high-quality service

User-friendly online submission system

Fair and swift peer-review system

Efficient typesetting and proofreading procedure

Display of the result of downloads and visits, as well as the number of cited articles Maximum dissemination of your research work

Submit your manuscript at: http://papersubmission.scirp.org/

Or contact jsip@scirp.org 